\title{
Efficient vibrational and translational excitations of a solid metal surface: State-to-state time-of-flight measurements of $\mathrm{HCl}(v=2, J=1)$ scattering from $\mathrm{Au}(111)$
}

\author{
Igor Rahinov, ${ }^{1}$ Russell Cooper, ${ }^{1}$ Cheng Yuan, ${ }^{2}$ Xueming Yang, ${ }^{2}$ Daniel J. Auerbach, ${ }^{3}$ and \\ Alec M. Wodtke ${ }^{1, a)}$ \\ ${ }^{1}$ Department of Chemistry and Biochemistry, University of California, Santa Barbara, \\ California 93106, USA \\ ${ }^{2}$ Dalian Institute of Chemical Physics, CAS, 457 Zhongshan Rd., Dalian, Liaoning 116023, \\ People's Republic of China \\ ${ }^{3}$ Gas Reaction Technologies, 861 Ward Dr., Santa Barbara, California 93111, USA
}

(Received 31 July 2008; accepted 28 October 2008; published online 4 December 2008)

\begin{abstract}
We report high resolution state-to-state time-of-flight (TOF) measurements for scattering of $\mathrm{HCl}(v=2, \quad J=1)$ from a $\mathrm{Au}(111)$ single crystal surface for both vibrationally elastic $(v=2 \rightarrow 2)$ as well as inelastic $(v=2 \rightarrow 1)$ channels at seven incidence energies between 0.28 and $1.27 \mathrm{eV}$. The dependences of the TOF results on final $\mathrm{HCl}$ rotational state and surface temperature are also reported. The translational energy transferred to the surface depends linearly on incidence energy and is close to the single surface-atom impulse (Baule) limit over the entire range of incidence energies studied. The probability of vibrational relaxation is also large. For molecules that relax from $v=2$ to $v=1$, the fraction of vibrational energy that is transferred to the surface is approximately $74 \%$. We discuss these observations in terms of an impulse approximation as well as the possible role of translational and vibrational excitations of electron-hole pairs in the solid.
\end{abstract}

(C) 2008 American Institute of Physics. [DOI: 10.1063/1.3028542]

\section{INTRODUCTION}

A fundamental understanding of the dynamics by which energy is exchanged at the gas-surface interface is a prerequisite to a comprehensive theory of gas-surface chemistry. Energy exchange involves translational, rotational, and vibrational degrees of freedom of the gaseous molecule and both phonon and electronic degrees of freedom of the solid. ${ }^{1}$ We can determine the total energy transferred to or from the solid by making beams of molecules with a well defined velocity and a specific internal state, scattering these from the surface of interest, and then using internal state and velocity resolved measurements of the scattered molecules. In this way, the total internal and translational energies lost from or gained by the molecule can be determined, from which one may derive the total energy gained or lost by the solid. There have been a limited number of state resolved experimental studies providing insight into the dynamics of vibrational relaxation and excitation during collisions of small molecules with metals and insulators; ${ }^{1-7}$ furthermore, many of the studies performed so far lacked sufficient translational resolution to properly quantify the interplay between the changes in the rovibrational energy, changes in the translational energy, and energy transfer to the solid. Even ignoring these limitations, the number of systems studied so far is quite limited. This lack of accurate experimental knowledge of translational energy is unfortunate as establishing the role

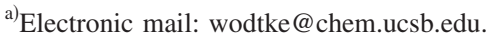

of translation is essential to characterizing the basic nature of alternatively proposed mechanisms for molecular energy transfer at surfaces. ${ }^{8}$

For electronically adiabatic energy transfer mechanisms, translation $(T)$ is expected to excite both phonons $(\phi)$ of the solid $^{8,9}$ as well as supply much of the required energy for vibrational $(V)$ and rotational $(R)$ excitations $^{10}$ of the colliding molecule. We refer to this as $T-V, R, \phi$ energy transfer. For an electronically adiabatic energy transfer mechanism, vibrational relaxation would be expected to channel a substantial amount of vibrational energy into translation $(V-T)$ of the scattered molecule.

The dominant coupling channels are quite different for electronically nonadiabatic energy transfer. In that case present models suggest that molecular vibration $(V)$ may be directly coupled to electronic $(E)$ degrees of freedom of the solid. Here, translation can and does enhance vibrational excitation, ${ }^{3,11}$ but this is thought to be due to an increase in the $V$-E coupling strength induced by penetration of the molecule into regions of higher electron density for higher energy collisions. ${ }^{3,12}$ Measurements of the vibrational excitation versus normal translational energy show "zerotranslational-energy thresholds," which have been interpreted as indicative of direct $E-V$ coupling. ${ }^{3}$ These results suggest that for vibrational relaxation, conversion of vibrational to translational energy would be small.

This "spectator view" for electronically nonadiabatic vibrational energy transfer, while widely discussed, has not been experimentally confirmed with sufficient accuracy. For multiquantum vibrational relaxation of $\mathrm{NO}(v=15)$ in colli- 
sions with $\mathrm{Au}(111)$, no excess translational energy in the scattered molecules could be observed even for loss of many vibrational quanta; however, one must hasten to add that the translational energy resolution in that study was not optimized. ${ }^{5}$ Analogous experiments designed to reveal the translational recoil of $\mathrm{H}(\mathrm{D})_{2}(v=0)$ produced in vibrational relaxation of $\mathrm{H}(\mathrm{D})_{2}(v=1)$ from copper have also been reported. $^{13-16}$ Here vibrational relaxation channels were found with substantially higher translational energies than the incident beam. ${ }^{13,14}$ Since the mechanism of vibrational relaxation is still controversial, ${ }^{16,17}$ it is unclear whether this is evidence against the spectator view of electronically nonadiabatic energy transfer or rather reflects electronically adiabatic dynamics.

State resolved measurements of $\mathrm{HCl}$ scattering from $\mathrm{Au}(111)$ have been previously reported..$^{9,11,18}$ In one of those studies, time-of-flight (TOF) experiments on translational inelasticity were reported and a direct scattering mechanism could be clearly demonstrated. ${ }^{18}$ But this work, as that of Ref. 5, was better optimized to obtaining state-to-state probabilities than to obtaining changes in the molecule's velocity distribution.

The interest of this paper is also the dynamics of $\mathrm{HCl}$ collisions with a $\mathrm{Au}(111)$ surface, where we have focused on the role of translational motion in molecule surface energy transfer. We emphasize two dynamical questions in this paper: (1) the nature of surface excitation by $\mathrm{HCl}$ translation and (2) the degree of coupling of translation in vibrational relaxation. This second point is an attempt to evaluate the "spectator view of translation" in electronically nonadiabatic vibrational energy transfer.

The experiment is designed to obtain much better translational energy resolution than prior state-to-state studies. Infrared overtone pumping $(v=0 \rightarrow 2)$ is combined with statespecific detection using resonantly enhanced multiphoton ionization (REMPI) in a favorable excitation-detection geometry to provide high resolution translational energy distributions for rovibrationally resolved state-to-state scattering channels. We obtain data for both vibrationally elastic $(v$ $=2 \rightarrow 2)$ and inelastic $(v=2 \rightarrow 1)$ scatterings over a wide range $(0.28-1.27 \mathrm{eV})$ of incidence energies, at several surface temperatures. In addition, we present a preliminary study of the rotational systematics of the scattering.

We find that the translational inelasticities in both vibrationally elastic $(v=2 \rightarrow 2)$ and inelastic $(v=2 \rightarrow 1)$ channels are large at all incidence energies, approaching the largest energy transfer possible for an impulsive collision with a single surface atom. One possible explanation of this large energy transfer is that translational excitation of electronhole pairs plays a significant role in these experiments. Additionally, we find that the average translational energy of scattered $\mathrm{HCl}$ is slightly larger for the $(v=2 \rightarrow 1)$ channel in comparison to the $(v=2 \rightarrow 2)$ channel. While this indicates that the spectator view of translation in electronically nonadiabatic vibrational energy transfer described above is qualitatively correct, the finite excess translational energy release in the $(v=2 \rightarrow 1)$ channel suggests that more complex dynamics are involved.

\section{EXPERIMENTAL}

The experimental apparatus employed in this work has been described in detail previously. ${ }^{19}$ In summary, short supersonic molecular beam pulses $[\sim 50 \mu$ s full width at half maximum (FWHM)] are generated by expanding a carrier gas seeded with $\mathrm{HCl}$ at $\sim 3$ atm stagnation pressure through a piezoelectrically actuated pulsed valve. The use of $\mathrm{H}_{2}, \mathrm{~N}_{2}$, and $\mathrm{D}_{2}$ in different mixing ratios as a carrier gas and varying concentrations of $\mathrm{HCl}$ (from $1 \%$ to $10 \%$ ) allowed us to obtain molecular beams with seven different incidence energies of translation in the range from 0.28 to $1.27 \mathrm{eV}$. The skimmed molecular beams pass through two differential pumping stages into an UHV surface science chamber, where they collide with a $\mathrm{Au}(111)$ crystal at an incidence angle of $\sim 3^{\circ}$ with respect to the surface normal. We were able to vary the temperature of the gold crystal with the aid of resistive heating and conductive cooling by liquid nitrogen. The base pressure in the surface chamber is $\sim 1 \times 10^{-10}$ Torr and reaches $\sim 1 \times 10^{-9}$ Torr with the pulsed molecular beam running at $10 \mathrm{~Hz}$. The surface was cleaned daily by sputtering with $3 \mathrm{kV} \mathrm{Ar}^{+}$for $\sim 50 \mathrm{~min}$ and then annealed to $\sim 1000 \mathrm{~K}$ for $\sim 30 \mathrm{~min}$. The cleanliness of the $\mathrm{Au}(111)$ crystal is confirmed by using Auger electron spectroscopy. The main impurity seen before cleaning is carbon. Based on the Auger spectra we estimate the impurities to be below 3\% of surface coverage after sputtering. The surface chamber is equipped with optical access ports allowing transmission of IR and UV beams for state-specific preparation of $\mathrm{HCl}$ molecules in $v=2$ using infrared overtone pumping as well as $2+1$ REMPI detection.

IR light at $\lambda \sim 1.7 \mu \mathrm{m}$ was produced by difference frequency mixing in a beta barium borate (BBO) crystal. The output from a tunable dye laser (Sirah Cobra-Stretch®) operating on a mixture of DCM and LDS 698 dyes pumped by the second harmonic of a Nd:YAG (yttrium aluminum garnet) laser (Continuum 7010IS) was mixed with the residual $1064 \mathrm{~nm}$ output of the Nd:YAG laser. In this way, we produced $\sim 3 \mathrm{~mJ} /$ pulse of tunable IR with a bandwidth of $\sim 1 \mathrm{~cm}^{-1}$. Unfortunately, we had to turn off the injection seeder of the Nd:YAG laser to broaden the frequency distribution of the IR light to avoid spurious instabilities in pumping efficiency produced by the mode structure of the dye laser. The IR light was focused with a $f=50 \mathrm{~cm}$ lens to a waist of $\sim 100 \mu \mathrm{m}$ where it crossed the molecular beam. We estimate the excitation efficiency to be $\sim 10^{-3}$.

REMPI was performed using UV pulses generated with a tunable dye laser (Sirah Cobra-Stretch®) lasing with Coumarin 503 dye, pumped by the third harmonic of a second Nd:YAG laser (Continuum 7010). The output of the dye laser is frequency doubled in a $\mathrm{BBO}$ crystal and produced radiation at $\lambda \sim 250 \mathrm{~nm}$ (pulse energy of $\sim 4-6 \mathrm{~mJ}$ ) and focused with a $f=30 \mathrm{~cm}$ lens to a waist of $\sim 10 \mu \mathrm{m}$. The laser beam crosses the $\mathrm{HCl}$ molecular beam in such a way that the beam waist is displaced behind the molecular beam. This position is carefully adjusted to minimize nonresonant multiphoton ionization. The ions produced in this way are detected by an 

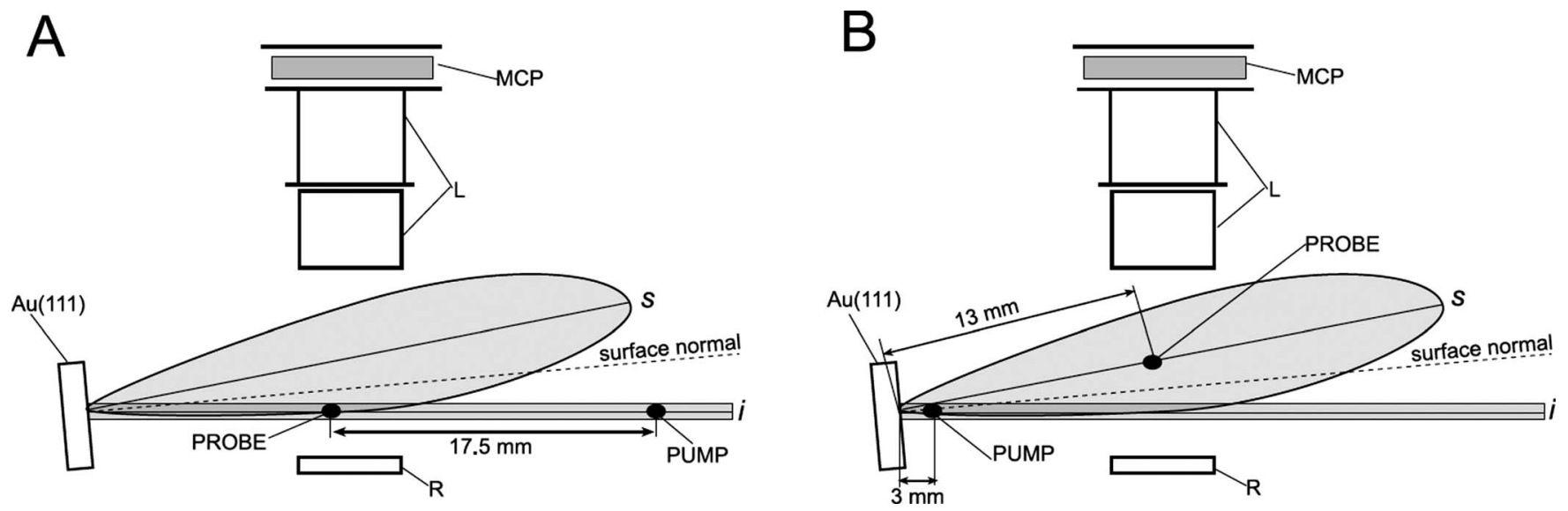

FIG. 1. Schematic of velocity measurements for the incident (a) and scattered (b) $\mathrm{HCl}$ molecules. s: scattered beam at the specular angle; i: incident beam; MCP: microchannel plate; L: focusing lens; R: repeller; pump: waist of IR beam (near $1.7 \mu \mathrm{m})$ pumping $\mathrm{HCl}$ from $(v=0, J=0)$ to $(v=2, \quad J=1)$; probe: waist of $2+1$ REMPI beam (near $250 \mathrm{~nm})$ probing the incident beam via $E^{1} \Sigma^{+}\left(v^{\prime}=0\right)-X^{1} \Sigma^{+}\left(v^{\prime \prime}=2\right)\left[Q(1)\right.$ transition] and the scattered beam via $E^{1} \Sigma^{+}\left(v^{\prime}\right.$ $=0)-X^{1} \Sigma^{+}\left(v^{\prime \prime}=2\right)$ and $E^{1} \Sigma^{+}\left(v^{\prime}=0\right)-X^{1} \Sigma^{+}\left(v^{\prime \prime}=1\right)[Q(0)-Q(9)$ transitions $]$.

ion collection system consisting of a repellor and an electrostatic focusing lens with two cylindrical elements, followed by a two-stage microchannel plate (MCP).

Figure 1 shows two experimental geometries used in these experiments. In Fig. 1(a), an IR (pump) pulse is used to excite $\mathrm{HCl}$ from $(v=0, J=0)$ to $(v=2, J=1)$ and the $\mathrm{UV}$ (probe) pulse is used to state specifically ionize those molecules $17.5 \mathrm{~mm}$ from the pump. By varying the time delay between the laser pulses, the velocity distribution of the molecular beam can be determined. Figure 1(b) shows the geometry used to detect $\mathrm{HCl}$ molecules in various quantum states that are produced by collisions of $\operatorname{HCl}(v=2, J=1)$ with a $\mathrm{Au}(111)$ surface. Here the molecules are pumped with IR light at distance of $3 \mathrm{~mm}$ (or less) from the $\mathrm{Au}(111)$ surface. The REMPI laser state specifically detects $\mathrm{HCl}$ at a distance of $13 \mathrm{~mm}$ from the surface. Note that in Fig. 1(b), the probe laser beam is shown to be offset vertically to detect molecules recoiling at the specular scattering angle. The laser beam can also be translated vertically to probe the scattering as a function of angle. The pump and probe laser beam positions are established by aligning the beams so they pass through removable apertures accurately positioned on the entrance and exit windows. The focusing lenses and steering optics are moved via micrometer driven stages to pass the beam through these apertures. Switching between the two geometries shown in Fig. 1 can be accomplished within about 5-10 $\mathrm{min}$ and the micrometers define the important geometric distances to better than $0.2 \mathrm{~mm}$. This uncertainty in distances results in a random error in inferring velocities from the TOF distributions $(\delta \ell / \ell=\delta t / t)$ on the order of $1 \%$.

\section{RESULTS}

Rotationally resolved IR-UV double resonance REMPI spectra of $\mathrm{HCl}$ in $v=2$ and 1 were recorded in several bands that have been previously reported. ${ }^{20}$ The incident molecular beams are rotationally cold, with the majority of the $\mathrm{HCl}$ molecules in the $(v=0, J=0)$ state. Overtone pumping prepares $\operatorname{HCl}(v=2)$ molecules exclusively in the $J=1$ rotational state.
Representative spectra obtained using the experimental geometry of Fig. 1(b) are shown in Fig. 2. Figure 2(a) shows the IR-UV double resonance spectrum in the vicinity of the $E^{1} \Sigma^{+}\left(v^{\prime}=0\right)-X^{1} \Sigma^{+}\left(v^{\prime \prime}=2\right)$ band origin. Lines in the $Q$-branch are indicated by the comb. Here, the UV REMPI laser fires about $12 \mu$ s after the IR laser and is sensitive only to molecules that have scattered from the surface. The laser time delay was adjusted to give maximum intensity, i.e., to the peak of the TOF distribution. Figure 2(b) shows a corresponding spectrum recorded with the IR laser off. The absence of any signal indicates that the assigned $Q$-branch spectral features appearing in Fig. 2(a) are exclusively due to rotationally inelastic scattering of $\operatorname{HCl}(v=2, J=1)$ with the $\mathrm{Au}(111)$ surface.

Figure 2(c) shows a similar spectrum in the vicinity of

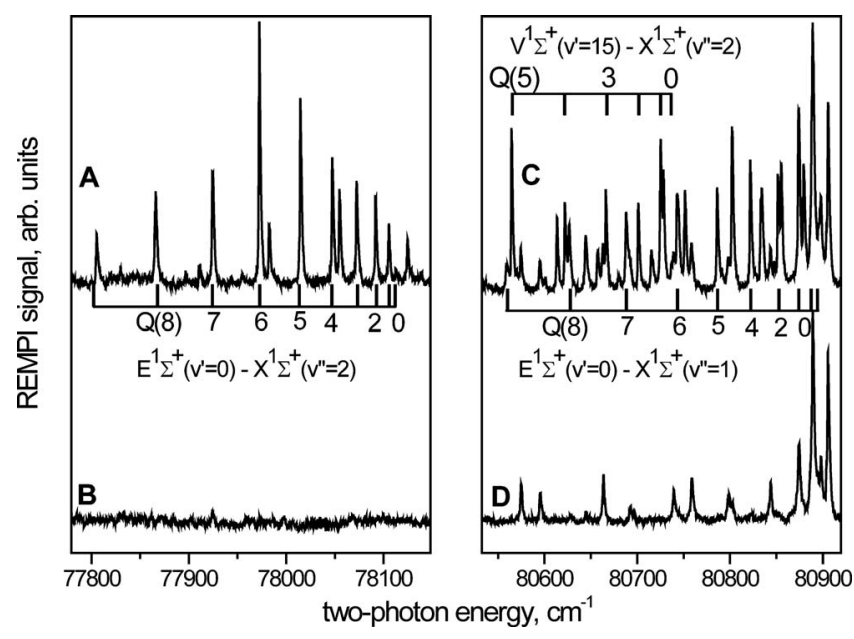

FIG. 2. 2+1 REMPI spectra for $\mathrm{HCl}$ scattered from $\mathrm{Au}(111)$. (a) and (c) were recorded with the IR pump laser on; (b) and (d) were recorded with IR pump laser off. The spectral range shown in (a) and (b) contains the $E^{1} \Sigma^{+}\left(v^{\prime}=0\right)-X^{1} \Sigma^{+}\left(v^{\prime \prime}=2\right)$ band ( $Q$-branch indicated by the comb) used for monitoring of $\operatorname{HCl}(v=2)$. The spectral range shown in (c) and (d) contains $E^{1} \Sigma^{+}(v=0)-X^{1} \Sigma^{+}\left(v^{\prime \prime}=1\right)$ transitions used for monitoring of $\operatorname{HCl}(v=1)$, as well as $V^{1} \Sigma^{+}\left(v^{\prime}=15\right)-X^{1} \Sigma^{+}\left(v^{\prime \prime}=2\right)$ transitions which overlap in this region ( $Q$-branches indicated by combs). The spectra were recorded for an incidence beam energy of $0.52 \mathrm{eV}$ and $300 \mathrm{~K}$ surface temperature. The delay between the two lasers was $\sim 12 \mu \mathrm{s}$. 


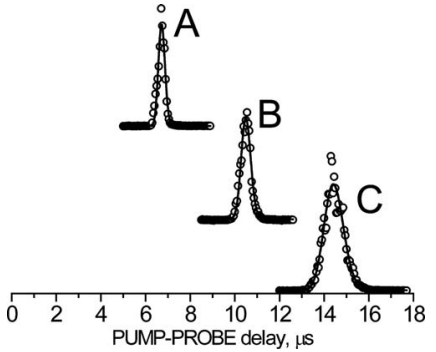

FIG. 3. Typical TOF spectra of incident $\operatorname{HCl}(v=2)$ measured by changing the delay between the IR pump pulse and the UV probe pulse. See text. (a) $1 \% \mathrm{HCl} / \mathrm{H}_{2}$ with $E_{I}=1.27 \mathrm{eV}$; (b) $10 \% \mathrm{HCl} / \mathrm{H}_{2}$ with $E_{I}=0.52 \mathrm{eV}$; (c) $10 \% \mathrm{HCl} / 20 \% \mathrm{~N}_{2} / 70 \% \mathrm{D}_{2}$ with $E_{I}=0.28 \mathrm{eV}$. The IR laser pumped the $R(0)$ line of the overtone band. The UV laser was tuned to probe the $E^{1} \Sigma^{+}\left(v^{\prime}\right.$ $=0)-X^{1} \Sigma^{+}\left(v^{\prime \prime}=2\right) Q(1)$ line. The open circles are experimental data points and the solid lines are fits to Eq. (A8)

the $E^{1} \Sigma^{+}\left(v^{\prime}=0\right)-X^{1} \Sigma^{+}\left(v^{\prime \prime}=1\right)$ band origin. Despite the spectrally coincident $V^{1} \Sigma^{+}\left(v^{\prime}=15\right)-X^{1} \Sigma^{+}\left(v^{\prime \prime}=2\right)$ band, which partially overlaps the $E^{1} \Sigma^{+}\left(v^{\prime}=0\right)-X^{1} \Sigma^{+}\left(v^{\prime \prime}=1\right)$ band here, one may still clearly identify the $Q$-branch of the $E^{1} \Sigma^{+}\left(v^{\prime}=0\right)-X^{1} \Sigma^{+}\left(v^{\prime \prime}=1\right)$ band. Figure 2(d) shows the same spectrum with the IR pump laser off. Weak spectral features arise from thermally populated $\operatorname{HCl}(v=1)$ in the molecular beam, some of which has undergone rotationally inelastic scattering from the $\mathrm{Au}(111)$ surface as well as some that has been produced by collisional excitation of $\mathrm{HCl}(v$ $=0 \rightarrow 1)$. This background is easily subtracted by comparing experiments with and without the IR pump laser.

For measurements of the incident beam's velocity distribution, the experimental geometry of Fig. 1(a) is used. By varying the time delay of the probe laser relative to the PUMP laser, TOF spectra of the incident $\operatorname{HCl}(v=2)$ molecules produced by overtone excitation are obtained. Typical TOF spectra of incident beams obtained in this fashion are shown in Fig. 3. The spread of arrival times and therefore the velocity spread in the incident beam, $\delta t / t=\delta v / v$, are found to be $6 \%-7 \%$. The beams were characterized by fitting the TOF data, after transformation to velocity space, to a function of the form

$$
F(v)=A v^{3} e^{-\left(\left(v-v_{0}\right) / \alpha\right)^{2}},
$$

where $A$ is a normalization constant, $v_{0}$ is a stream velocity, and $\alpha$ is a width parameter. The transformation procedure is described in Appendix. Table I shows the fitting results for the seven molecular beams used in this work.

TABLE I. Characteristics of the molecular beams used in this work.

\begin{tabular}{lcccc}
\hline \hline Mixing ratios & $\begin{array}{c}\left\langle E_{I}\right\rangle \\
(\mathrm{eV})\end{array}$ & $\begin{array}{c}\sqrt{\left\langle E_{I}^{2}\right\rangle-\left\langle E_{I}\right\rangle^{2} \mid} \\
(\mathrm{eV})\end{array}$ & $\begin{array}{c}\nu_{0}{ }^{\mathrm{a}} \\
(\mathrm{m} / \mathrm{s})\end{array}$ & $\begin{array}{c}\alpha^{\mathrm{a}} \\
(\mathrm{m} / \mathrm{s})\end{array}$ \\
\hline $10 \% \mathrm{HCl} / 20 \% \mathrm{~N}_{2} / 70 \% \mathrm{D}_{2}$ & 0.275 & 0.017 & 1210 & 52 \\
$10 \% \mathrm{HCl} / 10 \% \mathrm{~N}_{2} / 80 \% \mathrm{D}_{2}$ & 0.317 & 0.021 & 1297 & 60 \\
$10 \% \mathrm{HCl} / 90 \% \mathrm{D}_{2}$ & 0.447 & 0.027 & 1542 & 67 \\
$10 \% \mathrm{HCl} / 90 \% \mathrm{H}_{2}$ & 0.520 & 0.021 & 1665 & 48 \\
$5 \% \mathrm{HCl} / 95 \% \mathrm{H}_{2}$ & 0.779 & 0.062 & 2031 & 114 \\
$2.5 \% \mathrm{HCl} / 97.5 \% \mathrm{H}_{2}$ & 0.974 & 0.059 & 2276 & 98 \\
$1 \% \mathrm{HCl} / 99 \% \mathrm{H}_{2}$ & 1.268 & 0.056 & 2601 & 81 \\
\hline
\end{tabular}

${ }^{\mathrm{a}}$ The fitting parameters in this table refer to the following function: $F(v)$ $=A v^{3} e^{-\left(\left(v-v_{0}\right) / \alpha\right)^{2}}$.

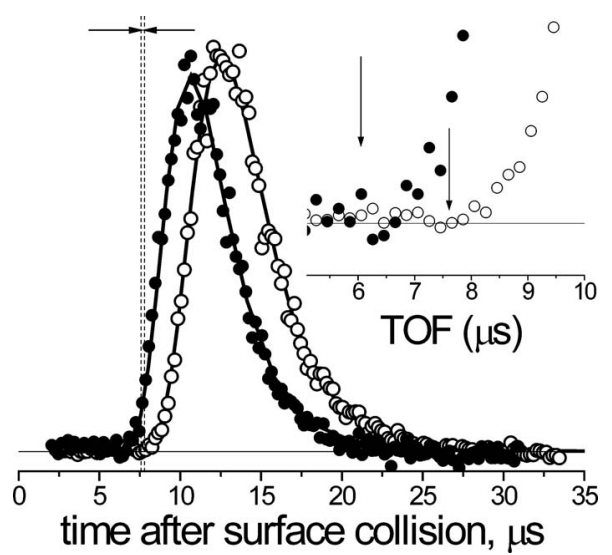

FIG. 4. TOF spectra of scattered $\operatorname{HCl}(v=2, J=5)$ (open circles) and $\operatorname{HCl}(v=1, J=5)$ (closed circles). The solid lines are fits using Eq. (A8). The scattered TOF spectra were recorded for an incidence beam energy of $\sim 0.52 \mathrm{eV}$ and surface temperature of $300 \mathrm{~K}$. Note that a hypothetical perfectly elastic process would reflect the velocity spread of the incident beam only and is indicated by vertical dashed lines. The arrows in the inset are the earliest possible arrival times, reflecting the energetic limits if the incidence energies of translational and vibrational were completely converted to final translational energy, assuming that the thermal energy of the surface is negligible.

The velocity spread inherent to the molecular beams method, while small, has two potentially deleterious effects on this work. First, the velocity spread limits to some degree the determination of the change in velocity induced by the collision with the gold surface. Second, and more importantly, the $\operatorname{HCl}(v=2)$ molecules spread out in space as they move away from the position where they are excited by the $100 \mu \mathrm{m}$ IR laser spot and hence the time at which the $\mathrm{HCl}(v=2)$ collides with the surface becomes less precisely defined as the distance between the IR laser and surface increases. By positioning the IR laser beam as close to the $\mathrm{Au}(111)$ surface as possible [Fig. 1(b)] this problem is minimized.

For the velocity spreads typical in this work $(6 \%-7 \%)$ the spatial extent of the prepared sample of $\operatorname{HCl}(v=2, J$ =1) spreads by $0.18 \mathrm{~mm}$, increasing from 0.1 to $0.28 \mathrm{~mm}$ over the pump-surface distance of $3 \mathrm{~mm}$; the effective resolution, $\delta t / t=\delta \ell / l=0.28 / 13=2.1 \%$, is slightly degraded for scattered molecules in comparison to measurements carried out on the incident molecular beam. This spread is still quite small compared to the spread of arrival times observed for molecules that scatter from the surface, a topic to which we now turn.

Figure 4 shows two typical TOF spectra for vibrationally elastic (open circles) and inelastic (closed circles) channels. The solid lines are the result of a fitting procedure described in Appendix using a function of the form of Eq. (A8). The two TOF spectra are shown with arbitrary (equal) peak intensities and do not indicate the relative branching between the two state-specific channels. Also shown (as vertical lines) is the range of arrival times expected for a hypothetical pureelastic process, indicating the arrival time spread expected due to the velocity uncertainty of the incident molecular beam. The observed broadening of the scattered molecules' arrival time distribution compared to the hypothetical elastic benchmark makes it clear that the observed shape of the TOF 


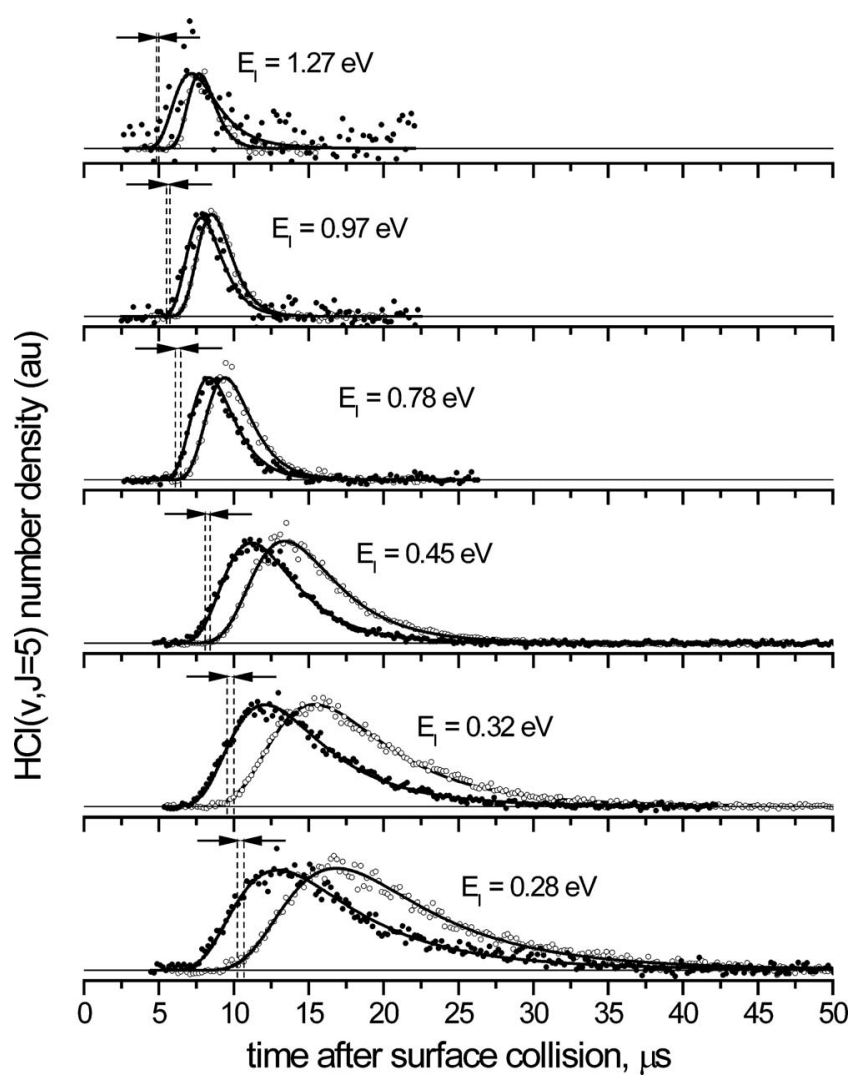

FIG. 5. Additional TOF data for vibrationally elastic and inelastic channels at kinetic energies of incidence from 0.28 to $1.27 \mathrm{eV}$ and surface temperature of 300 K. See Fig. 4 for additional details.

spectrum is dominated by the effects of inelastic scattering from the $\mathrm{Au}(111)$ surface. Considering the vibrationally elastic channel (open circles), one notes that the majority of observed signal arrives at times that indicate transfer of translation energy from the molecule to the surface. The short time (high energy) portion of the TOF spectrum is shown in the inset of Fig. 4. The arrows show the energetic limits for complete conversion of vibrational and translational energies of the incident $\mathrm{HCl}$ to outgoing translational energy. No signal is observed at these limits.

From Fig. 4, we see clearly that shorter arrival times are observed for the vibrationally inelastic $(v=2 \rightarrow 1)$ channel than for vibrationally elastic $(v=2 \rightarrow 2)$ channel. This result is consistent with the release of a portion of the vibrational energy to outgoing translational energy of the $\mathrm{HCl}$ molecule in the vibrational relaxation process and demonstrates that the view considering the translational motion to be a spectator to vibrationally inelastic scattering discussed in the introduction is not fully correct. The shift in time, however, is small and, as we will show in Sec. III A, represents conversion of only a small fraction of the available vibrational energy into translational energy.

Figure 5 shows additional data similar to that of Fig. 4 at a variety of incidence energies from 0.28 to $1.27 \mathrm{eV}$. The outgoing translational energy increases with incidence energy, which is a clear evidence for a direct scattering mechanism. In all cases, the vibrational relaxation channel exhibits slightly earlier arrival times than the vibrationally elastic channel. As was done in Fig. 4, the relative intensities for the

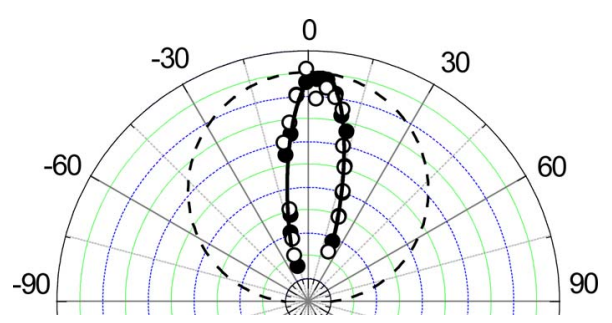

FIG. 6. (Color online) Angular distributions of product scattering channels resulting from incidence energy $\left\langle E_{I}\right\rangle=0.52 \mathrm{eV} \mathrm{HCl}(v=2, J=1)$ at $T_{S}$ $=300 \mathrm{~K}$. Vibrationally elastic channel, $\operatorname{HCl}(v=2, J=5)$ : open circles; vibrationally inelastic channel, $\operatorname{HCl}(v=1, J=5)$ : closed circles. Also shown are $\cos ^{28}\left(\theta-\theta_{0}\right)$, where $\theta_{0}=3^{\circ}$ (solid line) and $\cos (\theta)$ (dashed line).

two channels are arbitrarily scaled to equal peak intensity. In all cases the signals originating from $v=1$ and 2 are actually of comparable intensity, indicating that vibrational relaxation has a significant probability, probably $>0.1$. To obtain quantitative vibrational branching ratios, we would need additional data including rotational state distributions, angular distributions, and detection sensitivity for the two channels. Results on vibrational survival probability and branching ratios will be reported in a future publication.

Angular distributions of the incident and scattered $\mathrm{HCl}$ molecules were measured $\left(E_{I}=0.52 \mathrm{eV}\right.$ and $\left.T_{S}=300 \mathrm{~K}\right)$ by tuning the probe laser to specific transitions and translating the probe laser beam vertically, sweeping out a plane perpendicular to molecular beam direction. Figure 6 shows angular distributions for vibrationally elastic (open circles) and inelastic (closed circles) channels in the form of a polar plot. Both peak at approximately the specular angle and exhibit a FWHM of less than $30^{\circ}$. The solid line passing through the data points is a function of the form $\cos ^{28}\left(\theta-\theta_{0}\right)$, where $\theta$ is the exit angle with respect to the surface normal and $\theta_{0}=3^{\circ}$ is the specular angle. The angular distributions of scattered molecules also provide clear evidence that both vibrational channels result from a direct scattering mechanism.

\section{A. Translational energy release for $\mathrm{HCl}(v=2, J=1)$ $\rightarrow \mathrm{HCl}(v=2,1, J=5), T_{S}=300 \mathrm{~K}$}

Using the translational energy probability distribution for the incident and scattered molecules obtained in a way described in Appendix, we can calculate various moments of the energy distribution.

$$
\left\langle E_{S}^{n}\right\rangle=\int_{0}^{\infty} E^{n} P(E) d E .
$$

Results for the mean energy $\left\langle E_{S}\right\rangle$ and variance, $\sqrt{\left|\left\langle E_{S}^{2}\right\rangle-\left\langle E_{S}\right\rangle^{2}\right|}$, which reflects the width of the translational energy distribution, are given in Tables I-III for the incident beam, vibrationally elastic scattering, and vibrationally inelastic channels respectively. Tables I-III also show the fitting parameters needed to generate the analytical functions that accurately describe the velocity distributions of incident and scattered $\mathrm{HCl}$ observed in this work.

Typical results from the derived translational energy probability distributions are depicted in Fig. 7. Here, the experimentally derived distributions for vibrationally elastic (open circles) and inelastic channels (closed circles) are com- 
TABLE II. Characteristics of the velocity distributions of the vibrationally elastically scattered $\mathrm{HCl}$ observed in this work $(v=2 \rightarrow 2, \quad J=1 \rightarrow 5), T_{S}=300 \mathrm{~K}$. (data represented with open circles in Fig. 8).

\begin{tabular}{lcccccc}
\hline \hline Mixing ratios of incident beam & $\begin{array}{c}\left\langle E_{I}\right\rangle \\
(\mathrm{eV})\end{array}$ & $\begin{array}{c}\left\langle E_{S}\right\rangle \\
(\mathrm{eV})\end{array}$ & $\begin{array}{c}\sqrt{\left|\left\langle E_{I}^{2}\right\rangle-\left\langle E_{I}\right\rangle^{2}\right|} \\
(\mathrm{eV})\end{array}$ & $\begin{array}{c}\nu_{0}^{\mathrm{a}} \\
(\mathrm{m} / \mathrm{s})\end{array}$ & $\begin{array}{c}\alpha^{\mathrm{a}} \\
(\mathrm{m} / \mathrm{s})\end{array}$ & $\begin{array}{c}\text { fxn }^{\mathrm{b}} \text { sub-Baule } \\
(\%)\end{array}$ \\
\hline $10 \% \mathrm{HCl} / 20 \% \mathrm{~N}_{2} / 70 \% \mathrm{D}_{2}$ & 0.275 & 0.1126 & 0.0608 & 475 & 351 & 0.5 \\
$10 \% \mathrm{HCl} / 10 \% \mathrm{~N}_{2} / 80 \% \mathrm{D}_{2}$ & 0.317 & 0.13485 & 0.0657 & 605 & 334 & 2 \\
$10 \% \mathrm{HCl} / 90 \% \mathrm{D}_{2}$ & 0.447 & 0.17131 & 0.0707 & 780 & 305 & 12 \\
$10 \% \mathrm{HCl} / 90 \% \mathrm{H}_{2}$ & 0.520 & 0.20261 & 0.0733 & 900 & 285 & 15 \\
$5 \% \mathrm{HCl} / 95 \% \mathrm{H}_{2}$ & 0.779 & 0.37067 & 0.1162 & 1272 & 327 & 15 \\
$2.5 \% \mathrm{HCl} / 97.5 \% \mathrm{H}_{2}$ & 0.974 & 0.44436 & 0.1180 & 1439 & 299 & 19 \\
$1 \% \mathrm{HCl} / 99 \% \mathrm{H}_{2}$ & 1.268 & 0.53535 & 0.1234 & 1609 & 282 & $36^{\mathrm{c}}$ \\
\hline \hline
\end{tabular}

${ }^{\mathrm{a}}$ The fitting parameters in this table refer to the following function: $F(v)=A v^{3} e^{-\left(\left(v-v_{0}\right) / \alpha\right)^{2}}$.

${ }^{\mathrm{b}}$ The fraction of the translational energy distribution that is below the attractive Baule limit. See text.

${ }^{c}$ This value should be considered approximate. This data set exhibited the lowest signal to noise ratio of all of our experiments, especially on the long arrival time portion of the experimental TOF.

pared to a thermal distribution at the surface temperature (dotted line), the translational energy distribution of the incident molecular beam (gray shaded), and the translational energy distribution of the vibrationally elastic channel shifted to higher energy by the $\operatorname{HCl}(v=2-1)$ energy difference (dashed line).

There are several points to notice. First, the experimentally derived translational energy distributions are more energetic than a Maxwell-Boltzmann translational energy distribution at the surface temperature. Again, this result is consistent with a direct scattering mechanism. Second, for both vibrationally elastic as well as inelastic channels, a large fraction of the incidence energy is missing, having been converted to excitations of the solid. Third, the translational energy distribution of the vibrationally inelastic channel is only slightly more energetic than that of the vibrationally elastic channel and it is also broader. Fourth, a perhaps naïve expectation that all of the vibrational energy release would appear as additional translation beyond that exhibited by the vibrationally elastic channel (dashed curve) is not even approximately fulfilled. Results like those presented in Fig. 7 were obtained for six additional incidence energies (see Figs. 8 and 9) and (for two incidence energies) at several surface temperatures between 200 and $1000 \mathrm{~K}$. In all cases, the four points just described also apply.

Figure 10 shows the mean translational energy of the vibrationally elastic (open circles) and inelastic (closed circles) channels as a function of the mean incidence energy, when $\operatorname{HCl}(v, J=5)$ is state selectively detected. Note that for a rotational quantum number change from $J=1$ (prepared) to $J=5$ (detected), the rotational energy change $\Delta E_{\mathrm{rot}}$ is only $0.0341 \mathrm{eV}$, much smaller than the observed translational energy loss. The solid and dash-dotted lines are linear fits to these quantities. The dashed line represents the naïve "complete $V-T$ " expectation described in the previous paragraph. The error bars displayed in the figure are based on the combined statistical uncertainty associated with the mean energy evaluation as well as the error associated with uncertainty of the distances between pump and probe laser beams and the gold crystal. It is apparent from Fig. 10 that the dependence of the mean translational energy of the scattered molecules on the incidence energy is well described by a linear fit both for the vibrationally elastic and inelastic channels. For the vibrationally elastic channel, $\left\langle E_{S}\right\rangle=0.44\left\langle E_{I}\right\rangle$, and for the vibrationally inelastic channel $\left\langle E_{S}\right\rangle=0.44\left\langle E_{I}\right\rangle+0.082$, where all energies are in $\mathrm{eV}$. The fact that the slopes of these two lines are both 0.44 indicates that for both the vibrationally elastic and inelastic scattering channels, $56 \%$ of the incidence energy is transferred to the surface. Furthermore, this efficient translational excitation of the solid extends over the entire range of incidence energies studied. The nonzero $y$-intercept $(0.082 \mathrm{eV})$ for the vibrationally inelastic channel corresponds to approximately $\sim 26 \%$ of the vibrational energy. This result is consistent with $\sim 26 \%$ of the vibrational energy

TABLE III. Characteristics of the velocity distributions of the vibrationally inelastically scattered $\mathrm{HCl}$ observed in this work $(v=2 \rightarrow 1, J=1 \rightarrow 5), T_{S}=300 \mathrm{~K}$. (data represented by closed circles in Fig. 8).

\begin{tabular}{lccccc}
\hline \hline Mixing ratios of incident beam & $\begin{array}{c}\left\langle E_{I}\right\rangle \\
(\mathrm{eV})\end{array}$ & $\begin{array}{c}\left\langle E_{S}\right\rangle \\
(\mathrm{eV})\end{array}$ & $\begin{array}{c}\sqrt{\left|\left\langle E_{I}^{2}\right\rangle-\left\langle E_{I}\right\rangle^{2}\right|} \\
(\mathrm{eV})\end{array}$ & $\begin{array}{c}\nu_{0}{ }^{\mathrm{a}} \\
(\mathrm{m} / \mathrm{s})\end{array}$ & $\begin{array}{c}\alpha^{\mathrm{a}} \\
(\mathrm{m} / \mathrm{s})\end{array}$ \\
\hline $10 \% \mathrm{HCl} / 20 \% \mathrm{~N}_{2} / 70 \% \mathrm{D}_{2}$ & 0.275 & 0.19183 & 0.1135 & 488 & 526 \\
$10 \% \mathrm{HCl} / 10 \% \mathrm{~N}_{2} / 80 \% \mathrm{D}_{2}$ & 0.317 & 0.21862 & 0.1102 & 737 & 445 \\
$10 \% \mathrm{HCl} / 90 \% \mathrm{D}_{2}$ & 0.447 & 0.24568 & 0.1036 & 922 & 375 \\
$10 \% \mathrm{HCl} / 90 \% \mathrm{H}_{2}$ & 0.520 & 0.27871 & 0.1148 & 995 & 389 \\
$5 \% \mathrm{HCl} / 95 \% \mathrm{H}_{2}$ & 0.779 & 0.47 & 0.1572 & 1408 & 396 \\
$2.5 \% \mathrm{HCl} / 97.5 \% \mathrm{H}_{2}$ & 0.974 & 0.52517 & 0.1548 & 1535 & 363 \\
$1 \% \mathrm{HCl} / 99 \% \mathrm{H}_{2}$ & 1.268 & 0.61473 & 0.2431 & 1511 & 549 \\
\hline \hline
\end{tabular}

${ }^{a}$ The fitting parameters in this table refer to the following function: $F(v)=A v^{3} e^{-\left(\left(v-v_{0}\right) / \alpha\right)^{2}}$. 


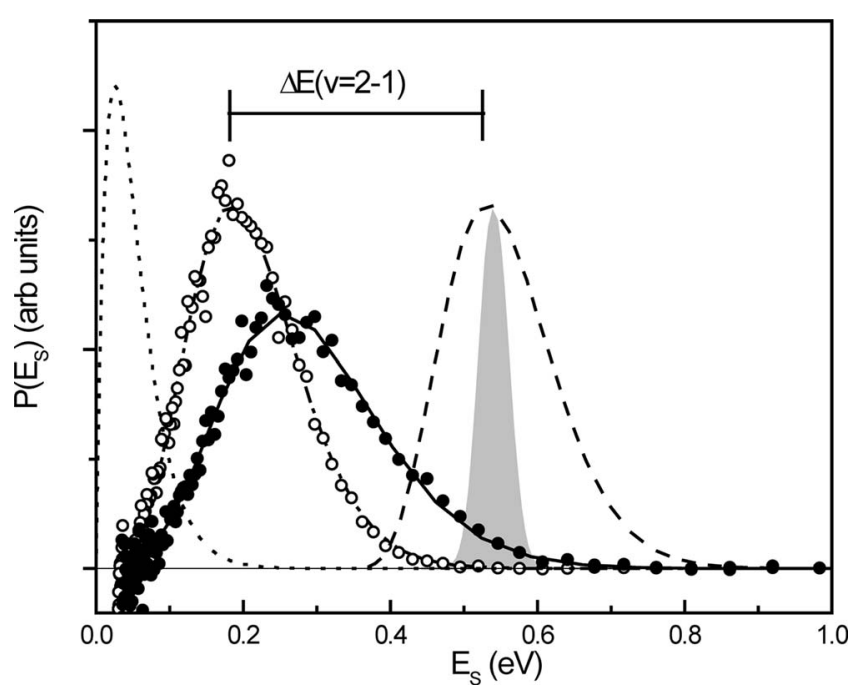

FIG. 7. Translational energy distributions for $\mathrm{HCl}$ scattering from $\mathrm{Au}(111)$. Derived translational energy distributions for recoiling $\operatorname{HCl}(v=2, J=5)$ (open circles) and $\operatorname{HCl}(v=1, J=5)$ (closed circles). The lines passing through the experimentally derived points are the results of the fitting analysis described in Appendix. The dashed line shows the $\operatorname{HCl}(v=2, J=5)$ results shifted to higher energy by the $\mathrm{HCl} v=2-1$ energy gap. The incident molecular beam translational energy distribution is shown in gray. A Maxwell-Boltzmann distribution at the surface temperature of $300 \mathrm{~K}$ is shown as a dotted line. The data presented here were recorded at incidence energy of $0.52 \mathrm{eV}$ and surface temperature of $300 \mathrm{~K}$.

being liberated to translation when $\mathrm{HCl}$ vibrationally relaxes in a $(v=2 \rightarrow 1)$ transition, independent of the incidence energy.

\section{B. Dependence of $\left\langle E_{S}\right\rangle$ on $\mathrm{HCl}$ rotation and surface temperature}

Much of the previous experimental works on inelastic scattering to which we might like to compare our results are for atom scattering from surfaces. Therefore, in comparing changes in translational energy for molecule scattering against work performed on atoms, it is important to characterize the dependence of $\left\langle E_{S}\right\rangle$ on the molecule's rotational quantum number. In particular, we are interested in two questions: (1) to what degree do the results of Fig. 10 depend on $J$ and (2) how different, if at all, is the rotationally elastic channel $\left(\mathrm{HCl}(v=2, J=1) \rightarrow \mathrm{HCl}\left(v^{\prime}, J=1\right)\right.$ from that shown in Fig. 10?

Although not the major emphasis of this work, we have obtained some data for other rotational states besides $J=5$. Figure 11 shows the average outgoing translational energy $\left\langle E_{S}\right\rangle$ for vibrationally elastic (open circles) and inelastic (closed circles) channels versus rotational excitation energy $\Delta E_{\text {rot }}$ for a range of final rotational states for $E_{I}=0.52 \mathrm{eV}$ and $T_{S}=300 \mathrm{~K}$. For both the vibrationally elastic and inelastic channels, the mean scattered translational energy decreases linearly with final rotational energy of the scattered $\mathrm{HCl}$. We will return to a more detailed discussion of these observations later in this paper. For now, it is most important to note that the range of overall rotational variation in $\left\langle E_{S}\right\rangle$ is small, less than $0.05 \mathrm{eV}$. More specifically, the difference in $\left\langle E_{S}\right\rangle$ for measurements on outgoing $J=5$ in comparison to

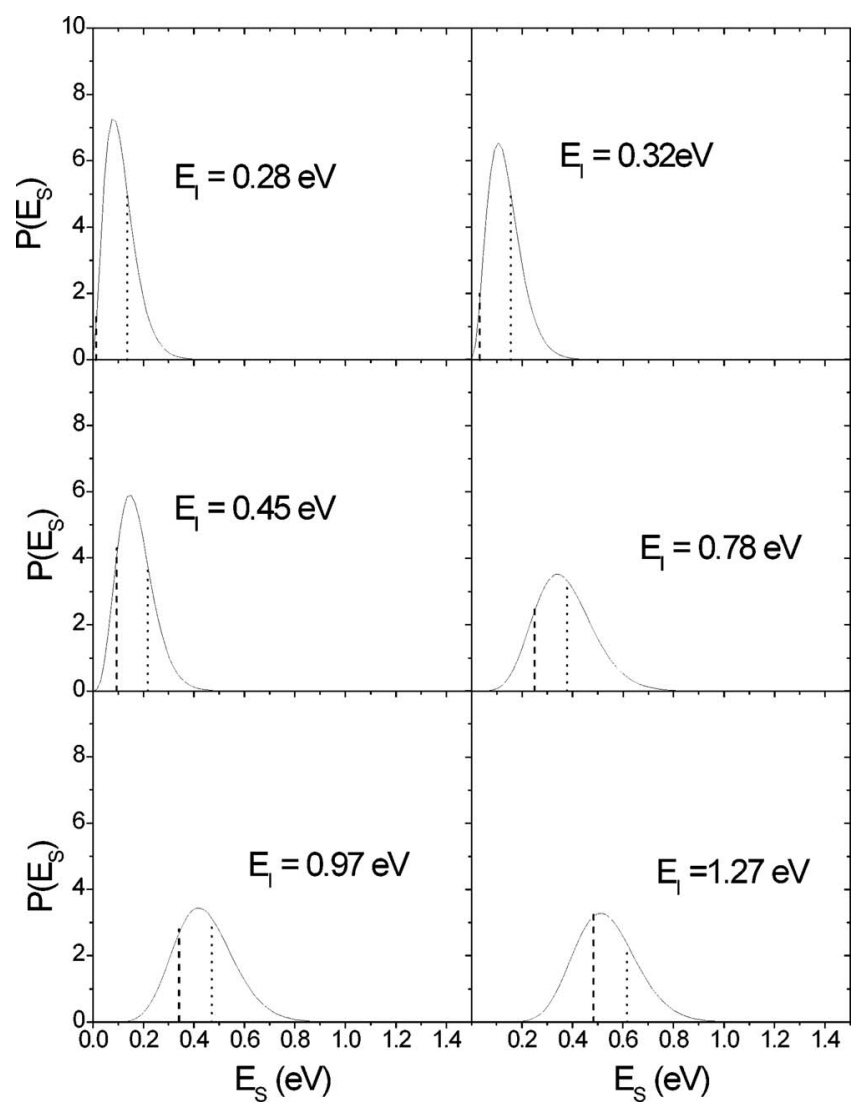

FIG. 8. Additional $\mathrm{HCl}$ translational energy distributions at various incidence energies for the $v=2-2$ channel. The vertical dashed line indicates the attractive Baule limit. The dotted line indicates the "Baule limit." The Baule limit is indicated by the solid line in Fig. 12. The attractive Baule limit is indicated as the dashed-dot line in Fig. 12, where the attractive well depth of $\mathrm{HCl}$ on $\mathrm{Au}(111)$ is assumed to be $0.23 \mathrm{eV}$ (Ref. 18). The attractive Baule limit is an estimation of the maximum translational inelasticity that can be obtained within a mechanical mechanism. See text. $T_{S}=300 \mathrm{~K}$. $\mathrm{HCl}$ was prepared in $v=2, J=1$ and detected in $v^{\prime}=2, J^{\prime}=5$.

$J=1$ - the rotationally elastic case that can be most naturally compared to atom scattering-is less than $0.025 \mathrm{eV}$, comparable to the error bars of Fig. 10.

During the molecule surface collision, energy transfer from the molecule to the solid may be accompanied by the reverse process, thermally populated phonon, and/or electron-hole pair de-excitation resulting in increased molecular excitation. For reasons of making simpler physical interpretations, it will be worthwhile to extrapolate our results to $T_{S}=0 \mathrm{~K}$, where only energy transfer from the molecule to the surface degrees of freedom is possible. Hence, it is important to understand how the results of Fig. 10 depend on $T_{S}$. We have examined the influence of $T_{S}$ on the mean scattered translational energy $\left\langle E_{S}\right\rangle$ for both the vibrationally elastic and inelastic channels for two values of the incidence energy, $E_{I}=0.52$ and $0.78 \mathrm{eV}$. The mean scattered translational energy shows a weak but unambiguous, positive linear dependence on surface temperature, exhibiting a stronger dependence at lower incidence energy, where the motion of the surface atom relative to the impinging molecule makes a more significant contribution.

For the surface temperature range from 200 to $1000 \mathrm{~K}$, the linear functions below accurately describe our observations. 


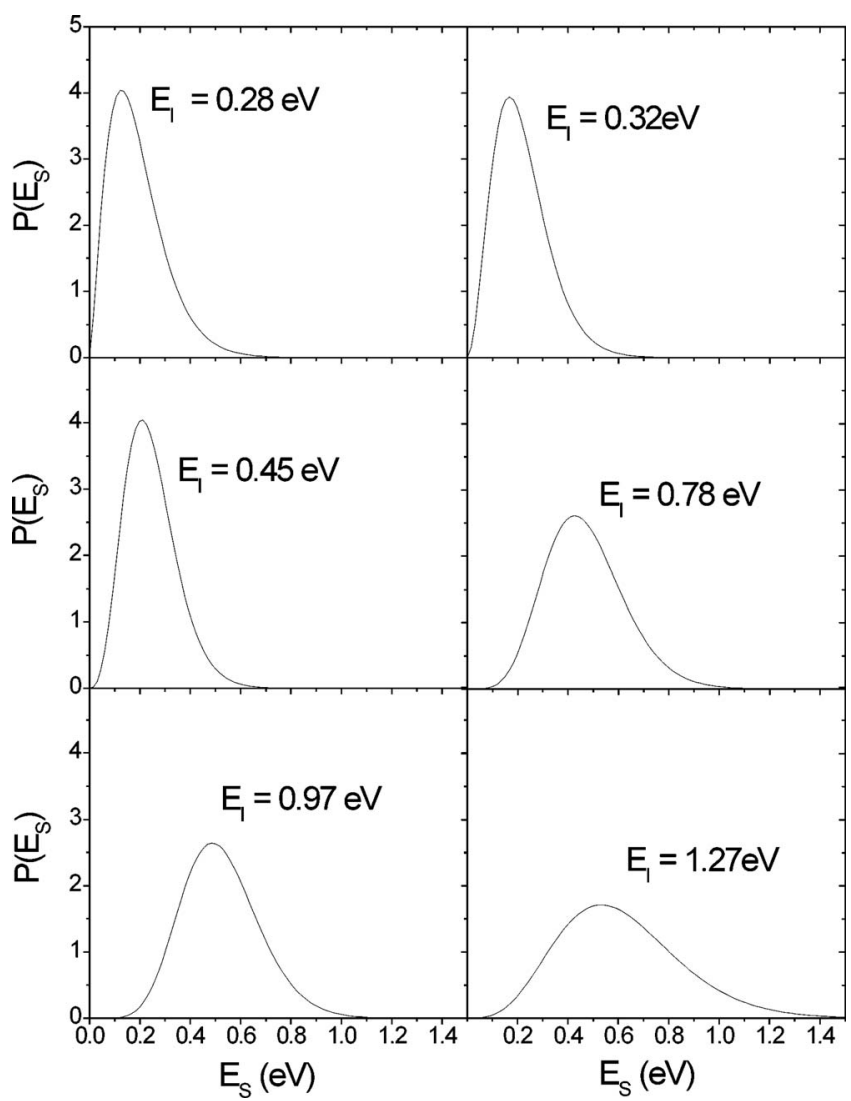

FIG. 9. Additional $\mathrm{HCl}$ translational energy distributions at various incidence energies for the $v=2-1$ channel. $T_{S}=300 \mathrm{~K}$. $\mathrm{HCl}$ was prepared in $v$ $=2, J=1$ and detected in $v^{\prime}=1, J^{\prime}=5$.

$$
\begin{aligned}
& \left\langle E_{S}\right\rangle=7.1_{ \pm 0.43} \times 10^{-5} T_{S}+0.257_{ \pm 0.002}, \\
& \operatorname{HCl}(v=2 \rightarrow 1, \quad J=1 \rightarrow 5), \quad E_{I}=0.52 \mathrm{eV}, \\
& \left\langle E_{S}\right\rangle=5.57_{ \pm 0.64} \times 10^{-5} T_{S}+0.185_{ \pm 0.004}, \\
& \operatorname{HCl}(v=2 \rightarrow 2, \quad J=1 \rightarrow 5), \quad E_{I}=0.52 \mathrm{eV}
\end{aligned}
$$

and

$$
\begin{aligned}
& \left\langle E_{S}\right\rangle=4.40_{ \pm 1.82} \times 10^{-5} T_{S}+0.461_{ \pm 0.007}, \\
& \operatorname{HCl}(v=2 \rightarrow 1, \quad J=1 \rightarrow 5), \quad E_{I}=0.78 \mathrm{eV}, \\
& \left\langle E_{S}\right\rangle=3.07_{ \pm 2.41} \times 10^{-5} T_{S}+0.369_{ \pm 0.012}, \\
& \operatorname{HCl}(v=2 \rightarrow 2, \quad J=1 \rightarrow 5), \quad E_{I}=0.78 \mathrm{eV} .
\end{aligned}
$$

The results represented by these equations show that the change in $\left\langle E_{S}\right\rangle$ resulting from cooling the surface from 300 to $0 \mathrm{~K}$ is about $0.02 \mathrm{eV}$, again a small effect and coincidentally comparable to rotational correction $(J=5$ to $J=1)$ to $\left\langle E_{S}\right\rangle$ just described, however, in the opposite direction.

To summarize this section on the rotational and surface temperature influences, the corrections involved in extrapolating our finite temperature results to the rotationally elastic case and to $0 \mathrm{~K}$ surface temperature are quite small in comparison to the translational energy loss. Moreover, the surface temperature and rotational extrapolations work in opposite directions approximately balancing one another. Thus,

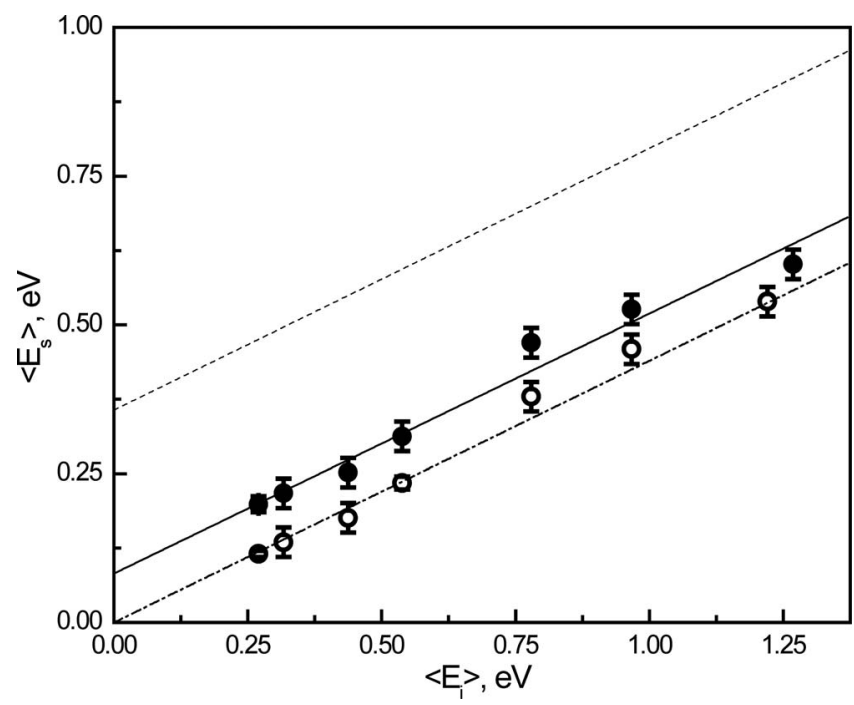

FIG. 10. Final translational energy for scattered $\mathrm{HCl}$. Vibrationally elastic $(v=2 \rightarrow 2)$ (open circles) and inelastic $(v=2 \rightarrow 1)$ (closed circles) are shown. The dash-dotted line is a linear fit for vibrationally elastic $(v=2 \rightarrow 2)$ channel- $\left\langle E_{S}\right\rangle=0.44\left\langle E_{I}\right\rangle$; the solid line is a linear fit for vibrationally inelastic $(v=2 \rightarrow 1)$ scattering- $\left\langle E_{S}\right\rangle=0.44\left\langle E_{I}\right\rangle+0.0821 \mathrm{eV}$. The dashed line represents the simplistic complete $V$ - $T$ expectation. All results shown here are for final $J=5$ and surface temperature of $300 \mathrm{~K}$.

we do not expect that the results of Fig. 10, taken at room temperature and for outgoing $\mathrm{HCl}$ in $J=5$, will deviate significantly from results at $T_{S}=0 \mathrm{~K}$ and $J=1$.

\section{DISCUSSION}

The most striking features of the experimental results presented are the following: (1) the amount of translational excitation of the solid is large over the entire energy range

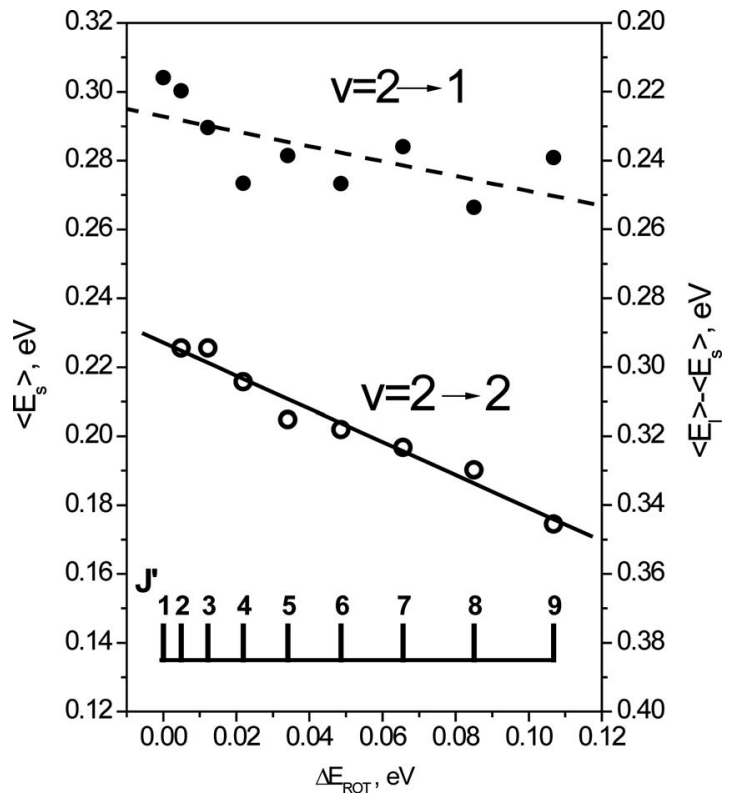

FIG. 11. Rotational anticorrelation with translational energy. The circles are derived form experimental data. The open circles represent the vibrationally elastic channel, while closed circles refer to the vibrationally inelastic channel. The linear fits to the data are empirical. See text. The comb shows the rotational quantum numbers of the scattered $\mathrm{HCl}\left(v^{\prime}, J^{\prime}\right)$ molecule. The incidence energy of translation was $0.52 \mathrm{eV}$ and the surface temperature was $300 \mathrm{~K}$. 
studied $\left(E_{I}=0.28-1.27 \mathrm{eV}\right) ;(2)$ the translational energy of scattered $\mathrm{HCl}$ is larger for the $v=2 \rightarrow 1$ channel in comparison to the $v=2 \rightarrow 2$ channel, but the energy difference is small compared to the vibrational spacing, a result that is approximately consistent with the spectator view of translation in electronically nonadiabatic vibrational energy transfer. We now explore these points in the context of past work.

\section{A. Vibrationally elastic $(v=2 \rightarrow 2)$ channel}

As presented in Sec. III A and in Fig. 10, for the vibrationally elastic channel the translational energy of the recoiling $\mathrm{HCl}$ is a constant fraction of the incidence energy, $\left\langle E_{S}\right\rangle$ $=0.44\left\langle E_{I}\right\rangle$, over the entire range of incidence energies studied $\left(E_{I}=0.28-1.27 \mathrm{eV}\right)$. According to the arguments of Sec. III $\mathrm{B}$, this relation is expected to be quantitatively accurate also for rotationally elastic scattering at $T_{S}=0 \mathrm{~K}$. This result naturally leads to the conclusion that on average a constant and large (0.56) fraction of the incidence energy is transferred to surface excitation when $\operatorname{HCl}(v=2)$ collides with the $\mathrm{Au}(111)$ surface for incidence energies between 0.28 and $1.27 \mathrm{eV}$.

An important question is whether such large translational inelasticity can be explained by purely mechanical energy transfer; that is, transferring $\mathrm{HCl}$ translation to $\mathrm{Au}$ atom kinetic energy. To understand this, let us consider our results in comparison to a simple model that represents the impulsive binary-collision limit, often referred to as the Baule limit. ${ }^{21}$ Here we imagine that the collision of a $\mathrm{HCl}$ molecule with a $\mathrm{Au}(111)$ surface happens in such a way that the $\mathrm{Au}-\mathrm{Au}$ interactions can be completely neglected, at least during the time of the $\mathrm{HCl}$-surface collision. We further approximate the $\mathrm{Au}-\mathrm{HCl}$ interaction as a zero impact parameter binary collision between a spherical projectile and a spherical Au surface atom. Such head-on collisions with zero impact parameter can transfer the greatest possible amount of translational energy from the $\mathrm{HCl}$ molecule to the $\mathrm{Au}$ atom. ${ }^{22}$ Note that this analysis is equivalent to using hard-cube model ${ }^{23}$ with the effective mass of the cube set to that of a single $\mathrm{Au}$ atom.

From linear momentum and total energy conservation in the collision pair, we may derive Eq. (2),

$$
\frac{E_{S}}{E_{I}}=\frac{\left(m_{1}-m_{2}\right)^{2}}{\left(m_{1}+m_{2}\right)^{2}} .
$$

For $\mathrm{HCl}$ colliding with $\mathrm{Au}, m_{1}=36$ and $m_{2}=196$, this leads to $E_{S}=0.475 E_{I}$. This compares well with the experimental result $\left\langle E_{S}\right\rangle=0.44\left\langle E_{I}\right\rangle$. Figure 12 shows the experimentally derived values of $\left\langle E_{S}\right\rangle$ for the vibrationally elastic channel plotted as a function of $\left\langle E_{I}\right\rangle$ compared to the prediction of Eq. (2), shown as a solid line. ${ }^{24}$ See also Fig. 8, where this energy is indicated by a vertical dotted line.

Within the context of the Baule model, a larger fraction of $E_{I}$ can be coupled to surface excitation due to the acceleration of the molecule by the attractive part of the interaction potential. If this attraction is modeled naïvely as a step function, the dashed-dot line of Fig. 12 obtains. See also Fig. 8 where this energy is indicated by a vertical dashed line. We call this dashed-dot line the "attractive Baule limit" in order

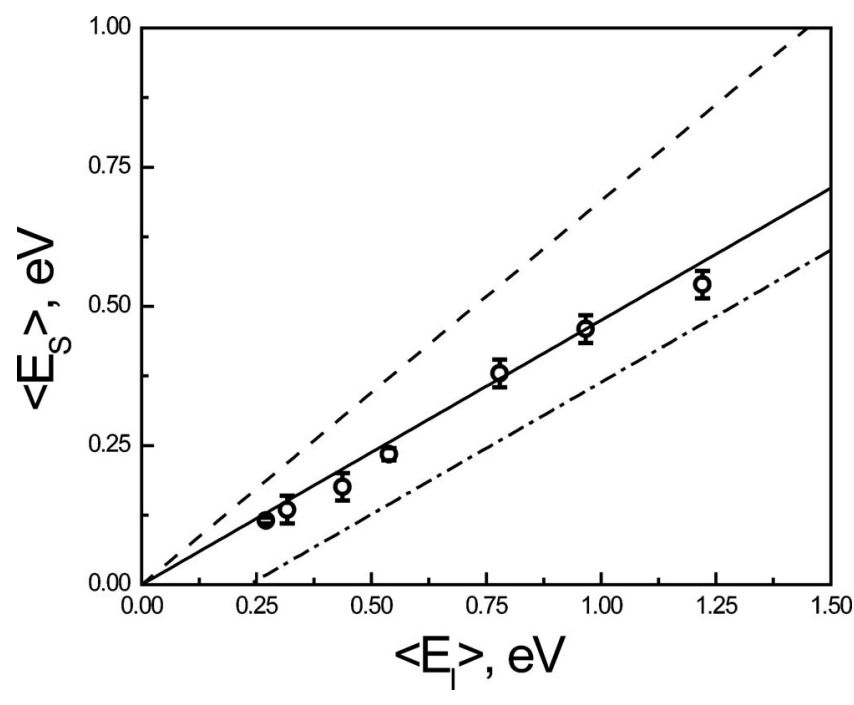

FIG. 12. Comparison to the binary-collision limit. Final translational energy for scattered $\mathrm{HCl}$ : vibrationally elastic $(v=2 \rightarrow 2)$ (open circles). All results shown here are for final $J=5$ and $T_{S}=300 \mathrm{~K}$. The solid line shows the translational recoil expected from a zero impact-parameter elastic collision of a $\mathrm{HCl}$ molecule with an isolated $\mathrm{Au}$ atom. The dashed line shows results expected by doubling the effective mass of the Au atom. The dashed-dot line is the attractive Baule limit, where the attractive well is treated as a step function (Ref. 22)

to distinguish it from the "simple Baule limit." Here an attractive well with a depth of $0.23 \mathrm{eV}$ has been employed, based on the orientation averaged binding energy reported by Lykke and Kay. ${ }^{18}$ The dashed-dot line does not describe the data. This could imply that the attractive energy between $\mathrm{HCl}$ and $\mathrm{Au}(111)$ is much smaller than $0.23 \mathrm{eV}$. Alternatively, Harris $^{22}$ showed that when the shape of the attractive well is modeled more realistically, for example, with a Morse function, the influence of the attractive well can be reduced and the results more closely resemble the simple Baule model with neglect of the potential well's influence.

In several previous examples of surface scattering in this energy range, the translational energy transferred to the surface is significantly less than the simple Baule limit. This has encouraged previous authors to define an effective mass of the surface atom in a hard-cube model, which is then larger than that of a single surface atom. In an empirical way, this is thought to reflect the nonbinary nature of the collision and the non-negligible forces between the surface atoms during the collision. The Baule equation-Eq. (2)—can be simply modified to express this,

$$
\left(\frac{E_{S}}{E_{I}}\right)_{N}=\frac{\left(m_{1}-N m_{2}\right)^{2}}{\left(m_{1}+N m_{2}\right)^{2}},
$$

where $N$ is a parameter representing the ratio of the effective hard-cube mass to the surface atom mass. This relation with $N=2$ is also shown in Fig. 12 as a dashed line and is clearly not in agreement with the observation.

Comparison of the results of this work to measurements of other scattering systems is complicated by the fact that many previous experiments were carried out at non-normal incidence angles, often $45^{\circ} .{ }^{18}$ For scattering from close packed faces of metals, the transfer of momentum perpendicular to the surface is much larger than that parallel to the 
TABLE IV. Translational inelasticity for several scattering systems.

\begin{tabular}{|c|c|c|c|}
\hline Scattering system & $\left\langle E_{S, \text { norm }}\right\rangle /\left\langle E_{I, \text { norm }}\right\rangle$ & Mass ratio $m_{1} / m_{2}$ & $N_{\text {surface atoms }}$ \\
\hline $\mathrm{Ar} / \mathrm{Pt}^{\mathrm{a}}$ & $0.6-0.7$ & 0.205 & $N_{\mathrm{Pt}_{\mathrm{t}}}=1.8-2.3$ \\
\hline $\operatorname{HCl}(v=2) / \mathrm{Au}^{\mathrm{b}}$ & 0.44 & 0.183 & $N_{\mathrm{Au}}=1$ \\
\hline $\mathrm{Ar} / \mathrm{Ag}^{\mathrm{c}}$ & $0.5-0.6$ & 0.370 & $N_{\mathrm{Ag}}=2.4-3.4$ \\
\hline $\mathrm{HCl}(v=2) / \mathrm{MgO}^{\mathrm{d}}$ & 0.40 & $2.25,1.5,0.9$ & $N_{\mathrm{O}}=10 ; N_{\mathrm{Mg}}=7 ; N_{\mathrm{MgO}}=4$ \\
\hline $\mathrm{H}_{2} / \mathrm{Cu}^{\mathrm{e}}$ & 0.95 & 0.031 & $N_{\mathrm{Cu}}=1-2$ \\
\hline $\mathrm{D}_{2} / \mathrm{Cu}{ }^{\mathrm{e}}$ & 0.875 & 0.063 & $N_{\mathrm{Cu}}=1-2$ \\
\hline $\mathrm{Ar} / \mathrm{C}^{\mathrm{f}}$ & 0.4 & 3.33 & $N_{\mathrm{C}}=5.8-8.3$ \\
\hline $\mathrm{Xe} / \mathrm{C}^{\mathrm{g}}$ & 0.14 & 10.9 & $N_{\mathrm{C}}=25$ \\
\hline $\mathrm{N}_{2} / \mathrm{Ru}^{\mathrm{h}}$ & $0.5-0.8$ & 0.277 & $N_{\mathrm{Ru}}=1.5-7$ (energy dependent) \\
\hline $\mathrm{NO} / \mathrm{Ag}^{\mathrm{i}}$ & $0.4-0.8$ & 0.278 & $N_{\mathrm{Ag}}=1-4$ (energy dependent) \\
\hline $\begin{array}{l}{ }^{\mathrm{a}} \text { Reference } 25 . \\
\mathrm{b} \text { This work. } \\
{ }^{\mathrm{c}} \text { Reference } 28 . \\
{ }^{\mathrm{d}} \text { Reference } 31 . \\
\text { e }{ }^{\mathrm{e}} \text { Reference } 32 .\end{array}$ & & $\begin{array}{l}{ }^{\mathrm{f}} \text { Reference } 29 . \\
{ }^{\mathrm{g}} \text { Reference } 30 . \\
{ }^{\mathrm{h}} \text { Reference } 7 . \\
{ }^{\mathrm{i}} \text { Reference } 6 .\end{array}$ & \\
\hline
\end{tabular}

surface. ${ }^{6,23,25,26}$ In the context of a hard-cube model, of course, parallel momentum is rigorously conserved. Thus, if we took the measured change in energy for an angle of incidence of $45^{\circ}$ on a low index plane of a metal, we would get an artificially low value of energy transferred when compared to the measurements made here at near normal $\left(\theta_{\mathrm{i}}\right.$ $=3^{\circ}$ ) incidence, since fully one-half of the incidence energy associated with motion parallel to the surface would be (at least approximately) conserved.

To avoid this difficulty, we have made comparisons of the energy associated with only the normal component of motion and derived an effective $N$ in Eq. (3) for each previous experiment that we are comparing with our results. Table IV presents a comparison of this sort. Similar surveys have been previously published where values of $N$ between about 2 and 4 are typical for application of the hard-cube and related models to data on inelastic scattering and trapping of atoms and molecules from solid surfaces. ${ }^{23,27} \mathrm{H}_{2} / \mathrm{D}_{2}$ scattering from $\mathrm{Cu}$ (Refs. 14 and 15) — one of the few state-to-state studies directly comparable to this work-exhibits quite significant energy transfer to the lattice (given the mass disparities), corresponding to $N=1-2$, although experimental uncertainties leave conclusive interpretations elusive. Scattering from insulators and graphite exhibits significantly larger values of $N$, reflecting the increased stiffness of these solids. One should note, however, that the application of the Baule model is not appropriate when the projectile outweighs the surface atom as chattering collisions may be important. 8,22

The Ar/Pt system ${ }^{25}$ is an interesting point of comparison with $\mathrm{HCl} / \mathrm{Au}$ because the masses of $\mathrm{Ar}$ and $\mathrm{Pt}$ are close to those of $\mathrm{HCl}$ and $\mathrm{Au}$, respectively, and because the quality of the data in that study is similar to that of the present work. Furthermore, those authors used an "isoflux contour" to decompose the energy transfer normal to the surface making comparison to the present work straightforward. For Ar/Pt, the approximate conservation of parallel momentum was explicitly verified at angles close to the peak in the angular distribution. Using high energy data for an incidence angle of $45^{\circ}$ and low surface temperature and extracting the energy transfer perpendicular to the surface, we find $N_{\mathrm{Ar}-\mathrm{Pt}}$
$=1.8-2.3$. Thus the energy transfer for $\mathrm{Ar} / \mathrm{Pt}$ is substantially less than that seen for $\mathrm{HCl} / \mathrm{Au}$, where we find $N_{\mathrm{HCl}-\mathrm{Au}}=1$. Quite similar to this is Ar scattering from Ag, which was studied in detail by Spruit et al. ${ }^{28}$ To account for the change in energy associated with normal motion to the surface observed in their work, ${ }^{28}$ one has to invoke an effective hardcube mass of 2.4-3.6 Ag atoms

Ar (Ref. 29) and Xe (Ref. 30) scattering from graphite has also been reported. Here angle-resolved incidence and scattered translational energy distributions were derived from high precision TOF measurements. Incidence energies were varied between 0.5 and $3.5 \mathrm{eV}$ for $\mathrm{Xe}$ and between 2.8 and $14.1 \mathrm{eV}$ for Ar and several incidence angles were used in both experiments. Conservation of parallel momentum was demonstrated in both studies. Analyzing the results within a hard-cube model, effective masses for the graphite surface of $70-100(N=6-8)$ for $\operatorname{Ar}$ (Ref. 29) and 300(N 25) for Xe (Ref. 30) were reported. Here, it is important to note that when the projectile (Xe or Ar) is heavier than the surface atom (C) a single bounce mechanism may not obtain as a single collinear impulsive collision between a moving $\mathrm{Xe}$ or Ar and stationary $\mathrm{C}$ is not sufficient to reverse the velocity of the projectile atom. Nevertheless, the derived value $N=25$ reflects a low efficiency for translational excitation of the solid in contrast to the present work.

Korolik et al. $^{31}$ studied vibrationally elastic $\operatorname{HCl}(v=2)$ scattering from $\mathrm{MgO}$ at near normal incidence and observed the fraction of the incidence energy retained in the scattered molecules. Here, there is some ambiguity as to the surface atom mass that is most meaningful within the context of the hard-cube model. We present calculations for three reasonable choices- $\mathrm{O}$ atom, $\mathrm{Mg}$ atom, and "MgO atom." In all cases the number of surface atoms is between 10 (O atoms) and 4 ( $\mathrm{MgO}$ atoms), showing substantially less translational inelasticity than for $\mathrm{HCl}$ on $\mathrm{Au}$.

$\mathrm{H}_{2}$ and $\mathrm{D}_{2}$ translational inelasticity in collisions with a $\mathrm{Cu}$ surface has also been measured using state-specific TOF methods like those presented here. ${ }^{32}$ Due to the large mass difference between $\mathrm{H}_{2}$ and $\mathrm{D}_{2}$ on the one hand and $\mathrm{Cu}$ on the other, the systematic errors in accounting for the translational inelasticity are large. That is, even for $N_{\mathrm{Cu}}=1$, the fractions 
of retained translational energy predicted by the hard-cube model are $88 \%$ for $\mathrm{H}_{2}$ and $78 \%$ for $\mathrm{D}_{2}$, which may be within the error limits of the reported results. ${ }^{32}$ Nevertheless, these authors report an effective mass of one to two $\mathrm{Cu}$ atoms.

One previous study gives results similar to this work at high incidence energies. Reference 6 reported high translational inelasticity for NO scattering from $\mathrm{Ag}(111)$, which depends strongly on the incidence energy. At $E_{I}=0.98 \mathrm{eV}$, translational inelasticity was consistent with a value of $N$ $=1$, similar to this work; but at lower values of $E_{I}$, the translational inelasticity was also reduced. For example at $E_{I}$ $=0.28 \mathrm{eV}$ a value of $N=4$ obtains. This is qualitatively similar to work where $N_{2}$ was scattered from Ru(0001). ${ }^{7}$ Here, the normal translational energy loss was measured for $E_{I}$ between 0.2 and $3 \mathrm{eV}$. While about $0.5 E_{I}$ was seen to be transferred to the surface at high incidence energies $(N$ as low as 1.4), this fraction decreased to less than or equal to $0.15 E_{I}(N \sim 7)$ at the lowest incidence energies studied. ${ }^{7}$

In contrast to all of these examples, the $\mathrm{HCl}$ on $\mathrm{Au}$ system is unique in its degree of translational inelasticity; that is, the observed energy transfer being at or beyond the binary-collision impulsive limit over the full range of incidence energies from 0.28 to $1.27 \mathrm{eV}$ as shown in Fig. 12 . Indeed, the agreement with the simplest of models (the Baule model neglecting attraction) is striking. We now consider this apparent agreement in more detail.

One possible interpretation of the result of this work is that the simple Baule model neglecting attraction accurately reflects the dynamics of $\mathrm{HCl}$ transitional excitation of $\mathrm{Au}(111)$. The validity of an impulse model is not obviously inappropriate to this system. The binary-collision impulsive limit can be valid when incidence energies are reached where collective $\mathrm{Au}$ atom motion occurs on a time scale much longer than the collision. We can make a rough estimate of the time scales involved combining knowledge of velocities and length scales of the collision as well as the phonon spectrum of $\mathrm{Au}(111)$. The lowest incidence velocities used in the present work are $v \sim 1000 \mathrm{~m} / \mathrm{s}$. We estimate the length scale of the collision as $\delta=\left[\left(R_{\mathrm{vdW}, \mathrm{Au}}+R_{\mathrm{vdWCl}}\right)-\left(R_{\mathrm{cov}, \mathrm{Au}}+R_{\mathrm{cov}, \mathrm{Cl}}\right)\right]$ $=0.87 \AA$, from canonical van der Waals and covalent bonding radii of $\mathrm{Au}$ and $\mathrm{Cl}^{33}$ This leads to a collision time scale of $\tau=2 \delta / v=1.5 \times 10^{-13}$ s. This may be compared with the inverse Debye frequency of the $\mathrm{Au}$ solid, $1 / \omega_{D}=2.9$ $\times 10^{-13} \mathrm{~s}$, which reflects the oscillation time of the highest frequency collective motion of the solid. The estimated ratio of time scales, $2 / \tau \omega_{D},{ }^{34}$ is only about a factor of 4 for experiments where the two time scales are closest. At the highest incidence energies, it increases to about 9. This analysis suggests that an impulse approximation might be reasonably accurate at least for part of the range of incidence energies studied here.

Beyond this, the modeled translational inelasticities of Fig. 12 rely on a hard-cube approximation (zero impact parameter) and a binary-collision assumption. The fact that our experiments probe mainly specular scattering at near normal incidence-backscattering geometry-may tend to emphasize collisions at small impact parameter. However, if corrugation of the interaction potential is small, backscattering can occur from any surface site. In this case the statistical likeli- hood to observe scattering from surface sites where multiple surface atoms interact with the $\mathrm{HCl}$ projectile (e.g., near hollow and bridging sites) would be higher than scattering solely from A-top sites. This is likely the origin of the increased values of $N$ seen in the scattering of rare gas atoms from close packed metal surfaces. See Table IV. There is no evidence to suggest that the potential surface corrugation of $\mathrm{HCl}$ on $\mathrm{Au}(111)$ is large. Within this context, accurate potential surface calculations would be very valuable.

What other explanations of the large translational inelasticity might be possible? Clearly the nature of potential energy surfaces (PESs) and lattice properties might be peculiar for $\mathrm{HCl}$ on $\mathrm{Au}(111)$, and in the absence of rigorous theoretical attempts to simulate the scattering, we should not rule out the possibility of a purely mechanical energy transfer mechanism.

Another possible explanation is that a significant fraction of the translational incidence energy is absorbed by electronic degrees of freedom, producing excited electron-hole pairs. By considering translational inelasticity surpassing the Baule limit, we may reach insights about this interpretation. As already mentioned the dashed-dot curve of Fig. 12 represents the attractive Baule limit, where the attractive well depth is most efficiently coupled along with the incidence energy to mechanical excitation of the surface. Molecules that emerge from the surface collision with even lower translational energies are quite difficult to understand within the framework of a mechanical model. We argue that they exceed the mechanical limit for translational inelasticity. If a significant fraction of the recoiling molecules observed in this work surpass the attractive Baule limit, we might become more convinced that electron-hole pair excitation is important.

The values of $\alpha$ and $v_{0}$ given in Table II can be used to obtain a functional form-Eq. (A9) in Appendix-of the translational energy distribution of recoiling $\mathrm{HCl}$ at all of the incidence energies of the work. These functions can be seen in Figs. 7 and 8 for the vibrationally elastic channel. We then integrate the distributions in the low energy range between zero and the energy defined by the attractive Baule limit. See the rightmost column in Table II. The fraction exhibiting translational inelasticity exceeding the attractive Baule limit is small at low incidence energy and grows at higher incidence energies to a value of at least 0.2. This observation is difficult to explain within a purely mechanical model. It seems to us that translational excitation of electron-hole pairs should be considered as a possible explanation.

We do not claim that this work is the first indication of the translational excitation of surface electron-hole pairs; however, the experimental evidence presented here represents an energy range significantly lower than previous studies. For example, coupling of incidence translation to electron-hole pairs in metals and semiconductors can occur at incidence energies of 3-10 eV. ${ }^{35}$ Remarkably, the present study suggests that such dynamics can persist to energies important to thermal collisions at modest temperatures and at incidence energies that are comparable to physisorption well depths. If this interpretation is correct, theoretical simula- 
tions of trapping of $\mathrm{HCl}$ on $\mathrm{Au}$ are likely to fail unless they accurately reflect electron-hole pair excitation by translation.

\section{B. Vibrationally inelastic $(v=2 \rightarrow 1)$ channel}

As mentioned in connection with the presentation of Fig. 5 in Sec. III, the observed signal strengths for scattered $\mathrm{HCl}(v=2)$ and $\mathrm{HCl}(v=1)$ molecules are comparable, indicating a vibrational branching fraction, $\operatorname{HCl}(v=1): \mathrm{HCl}(v=2)$, greater than 0.1. It is interesting to compare this observation to other examples of vibrational relaxation on insulators. For example, vibrational relaxation could not be observed for $\mathrm{NO}(v=1)$ scattering from $\operatorname{LiF}(001){ }^{36}$ More directly related to the present work, Korolik et al. ${ }^{31}$ were unable to observe vibrational relaxation in direct scattering of $\operatorname{HCl}(v=2 \rightarrow 1)$ in collisions with a $\mathrm{MgO}$ surface. In contrast, only when trapping desorption was the dominant scattering mechanism, a situation where the interaction time between the molecule and the surface is orders of magnitude longer than in direct scattering, could a weak $\operatorname{HCl}(v=1)$ signal be detected. In contrast, $\mathrm{HCl}(v=2$ and 1$)$ are easily seen in this work and exhibit the characteristics of a direct scattering mechanism. This result, which is similar to prior results on $\mathrm{NO}^{5}$ strongly suggests that vibrational to electron-hole pair excitation is responsible for the enhanced vibrational relaxation seen here.

\section{Kinetic energy of molecules undergoing $v=2 \rightarrow v$ $=1$ transitions}

Previous work on the kinetic energy of molecules undergoing vibrationally inelastic transitions through an electronically nonadiabatic mechanism did not reveal an observable change in kinetic energy associated with vibrationally inelastic scattering. For example, in experiments where multiquantum vibrational relaxation of $\mathrm{NO}(v=15)$ on $\mathrm{Au}(111)$ was observed, the transfer of the vibrational energy lost to the translation of outgoing NO molecules could not be observed, despite the large magnitude of the vibrational energy lost in the collision. ${ }^{5}$ In another study of NO scattering from $\mathrm{Ag}(111)$, where vibrational excitation of $\mathrm{NO}(v=0 \rightarrow 1)$ was seen, ${ }^{2}$ the TOF distributions of the scattered $\mathrm{NO}(v=0)$ and $\mathrm{NO}(v=1)$ were only slightly different. Furthermore, an increase in likelihood of vibrational excitation with increasing translational energy" was observed with a "zerotranslational-energy threshold." ${ }^{3}$ This should be compared to examples of vibrational excitation in surface collisions that take place by a mechanical mechanism, where vibrational excitation occurs only when the translational energy exceeds the vibrational excitation energy. ${ }^{9-11}$

These previous results suggest the following picture for electronically nonadiabatic vibrational energy transfer, which we label the translational spectator view. In short, the translational degree of freedom may influence the magnitude of the coupling between vibration and surface electrons, presumably influencing the precise geometry including molecule-surface distance that may be explored in the scattering trajectory. However, the conversion of translation to vibration or vibration to translation is unimportant for electronically nonadiabatic vibrational energy transfer at metal surfaces.
Here, we have the ability to test the translational spectator view with high experimental precision. This is due to our ability to measure vibrationally elastic $-\mathrm{HCl}(v=2 \rightarrow 2)-$ and inelastic $-\mathrm{HCl}(v=2 \rightarrow 1)-$ channels under identical experimental conditions. In this way one may disentangle translational inelasticity not associated with vibrational energy exchange from translational energy exchange with vibration.

Figure 10 shows these results. Here one can see the average translational energy retained in molecules scattered through both vibrationally elastic (open circles) and inelastic (closed circles) channels as a function of incidence energy. Empirically, we see that both of these quantities scale approximately linearly with incidence energy of translation (solid and dash-dotted lines). Also shown is a dashed line that represents a hypothetical situation where all of the vibrational energy released in the inelastic channel is converted to outgoing translation beyond the translational energy carried away in the vibrationally elastic channel. Clearly, this expectation is not confirmed by the experimental observations. Quite the contrary, the solid line fit to the inelastic data represents only about $26 \%$ of the vibrational energy appearing as translation. We conclude that the translational spectator view is approximately confirmed by this work.

This result provides further evidence that a mechanical mechanism is not important in vibrational relaxation of $\mathrm{HCl}(v=2)$ on $\mathrm{Au}(111)$. Consider that the Debye frequency of $\mathrm{Au}$ may be represented as a wavenumber, $\omega_{D}=\nu_{D} / c$ $=118 \mathrm{~cm}^{-1}$. This value is only $(1 / 15)$ th that of the $\operatorname{HCl}(v$ $=2-1$ ) energy gap. If we assume a mechanical model, conversion of $74 \%$ of the vibrational energy to phonons would imply that about 12 phonons would need to be excited in a direct scattering mechanism. Such high order multiphonon processes are not expected to be efficient. In contrast, this amount of energy could be used to excite a single electronhole pair.

We may also consider a more detailed physical interpretation of the results shown in Fig. 10. Beyond the conclusion that the translational spectator view approximately obtains, it is interesting to consider what might account for the finite (26\%) excess translational energy observed in the vibrationally inelastic channel, in comparison to the vibrationally elastic case.

One possibility is that there are both electronically adiabatic and nonadiabatic scattering events taking place in this experiment. That is, underlying the smooth and apparently single component of the $(v=2 \rightarrow 1)$ translational energy distribution shown in Fig. 7 (closed circles) there are, in fact, two contributing components, one electronically adiabatic and one nonadiabatic. Following that line of reason, we might expect a component with a similar energy distribution as the vibrationally elastic channel (nonadiabatic), exhibiting $100 \%$ dissipation of vibrational energy to the surface, and a second, in which a large fraction of the of the vibrational energy release would appear as additional translation beyond that exhibited by the vibrationally elastic channel. However, we consider this line of analysis dubious for at least two reasons. First, we do not observe even a hint of bimodality in any of the raw TOF data. Hence, there is no need to invoke 


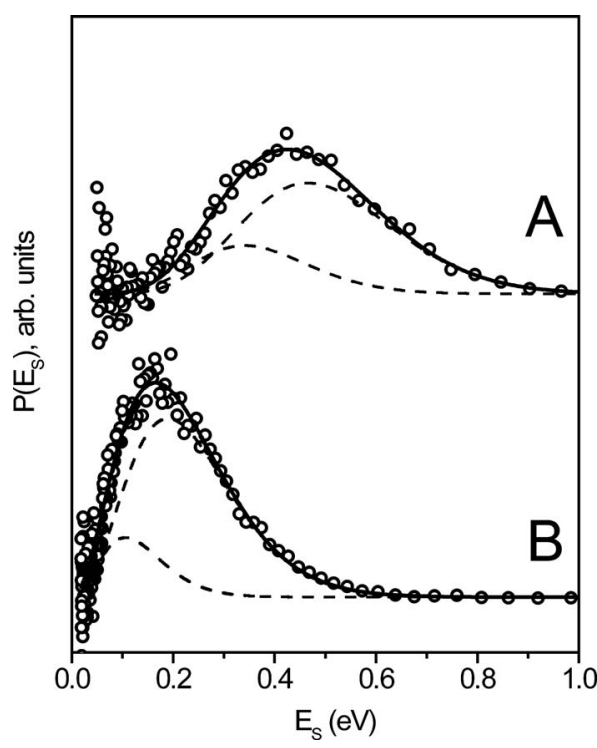

FIG. 13. An attempt to fit the $P\left(E_{S}\right)$ of scattered $\operatorname{HCl}(v=1, J=5)$ at $T_{S}$ $=300 \mathrm{~K}$ to two components. (a) Incidence energy of $0.78 \mathrm{eV}$; (b) incidence energy of $0.32 \mathrm{eV}$. The empty circles are experimentally derived energy distributions. The solid lines are fits to Eq. (4). The dashed lines depict the individual fast and slow components of the fit. See text.

a bimodal distribution to fit the observations of this work. Second, even when such a fitting procedure is pursued (as explained below), we find no evidence of a faster component consistent with what one would expect from a mechanical mechanism.

To demonstrate this we fitted the data like that in Fig. 7 to bimodal translational energy distributions described by

$$
\begin{aligned}
P(E)= & A_{1} \frac{E}{m^{2}} e^{-\left(2 / m \alpha_{1}^{2}\right)\left(\sqrt{E}-\sqrt{E_{01}}\right)^{2}} \\
& +A_{2} \frac{E}{m^{2}} e^{-\left(2 / m \alpha_{2}^{2}\right)\left(\sqrt{E}-\sqrt{E_{02}}\right)^{2}},
\end{aligned}
$$

where the values of $\alpha_{2}$ and $E_{02}$ are obtained from the fit of vibrationally elastic channels and are kept constant, while the relative amplitudes of two components $A_{1}$ and $A_{2}$ as well as the values defining the width and the position of the faster component $\alpha_{1}$ and $E_{01}$ are free parameters. Figure 13 displays examples of this analysis for two incidence energies. This "bimodal" fitting procedure results in a fast component that exhibits a mean scattered translational energy only $\sim 0.026 \mathrm{eV}$ higher than the values reported in Fig. 10, from a much simpler analysis fitting to a single distribution.

A second possible way to interpret the apparent $26 \%$ conversion of vibrational to translational energy in the vibrationally inelastic channel is much simpler; namely, that the translational spectator view of electronically nonadiabatic vibrational relaxation is only approximately true. This begs the question: what dynamics might be present that would lead to a noticeable translational energy release in electronically nonadiabatic vibrational relaxation. Here we can only speculate.

One possibility is that electronically nonadiabatic vibrational relaxation occurs only for specific angular orientations of the $\mathrm{HCl}$ molecule with respect to the surface or only for collisions at specific surface sites. If the collisions with these special orientations or at these special surface sites were coincidentally also taking place in such a way that translational inelasticity was substantially reduced in comparison to vibrationally elastic collisions, one could observe higher translational energies for vibrationally inelastic channels, without a direct coupling of vibrational to translational motion. One might imagine, for example, that collisions taking place at hollow sites, where the scattered molecule interacts simultaneously with three Au atoms, might be more translationally elastic than collisions that occur at A-top sites, where the scattering molecule interacts more intimately with a single $\mathrm{Au}$ atom.

This basic idea is not completely without precedent. For example, Kimman et al. ${ }^{37}$ studied rotational excitation of NO in collisions with $\operatorname{Ag}(111)$ and reported a decrease in the amount of energy transferred to the surface as the final rotational energy of the scattered molecule increased. The authors explained this observation by describing dynamics in which NO molecules approaching the surface with the proper orientation required for larger rotational excitation also experience reduced translational energy transfer to solid phonons. ${ }^{37}$ In another related study, Kroes and co-workers showed that $\mathrm{H}_{2}$ dissociation on copper occurs at different surface sites when considering different initial quantum states of $\mathrm{H}_{2} \cdot{ }^{38}$

One aspect of the observations of this work, however, speaks against this line of reasoning; the translational augmentation in the vibrationally inelastic channel (of about $0.08 \mathrm{eV}$ ) is independent of incidence energy. If an orientation or surface site specific influence is to be invoked, we must then explain how the reduction in translational inelasticity of $\sim 0.08 \mathrm{eV}$ is unchanged for values of $\left\langle E_{I}\right\rangle$ as large as 1.27 $\mathrm{eV}$ and as low as $0.28 \mathrm{eV}$. It appears to us more reasonable that if such a dynamics was indeed at work, it would result simply in a steeper dependence of $\left\langle E_{S}\right\rangle$ on $\left\langle E_{I}\right\rangle$ for the vibrationally inelastic channel in comparison to the vibrationally elastic channel. We conclude that this line of reasoning is not consistent with some of the observations of this work.

We suggest a possible alternative explanation for the translational augmentation observed for electronically nonadiabatic vibrational relaxation. All electronically nonadiabatic mechanisms of vibrational relaxation suggest a transient charge transfer (or at least partial charge transfer) from the metal to the molecule in the molecule's approach to the surface. Any such charge transfer will result in an attractive image-charge interaction between the molecule and the surface, which will indeed accelerate the molecule above its initial translational energy. If the charge transfer is sufficiently long lived to allow appreciable acceleration, one might easily postulate that the translation-to-surface energy transfer is similar to that seen in the vibrationally elastic channel, but that there is an effective charge transfer induced enhancement of the translational energy of incidence. In order for such a model to give rise to an energy independent augmentation to the $\left\langle E_{S}\right\rangle$, one would only need to define a distance at which the charge is transferred to the molecule, 
$z_{>}$, and a distance at which the charge is transferred back to the surface, $z_{<}$, as independent (or perhaps only weakly dependent) of incidence energy of translation.

Finally, we wish to comment on the results of vibrationally inelastic scattering on a related collision system $\left(\mathrm{H}_{2}\right.$ on $\mathrm{Cu}$ ), studied by Watts and Sitz. ${ }^{14}$ This experiment provides state resolved velocity measurements, and at least superficially, some of the observations of that work are similar to those reported here. In that work, $\mathrm{H}_{2}(v=1, J=1)$ was prepared by stimulated Raman pumping and state-to-state TOF on $\mathrm{H}_{2}(v=1$ or $0, J)$ data was obtained using REMPI. ${ }^{14}$ The vibrational survival probability declines with increasing incidence energy reaching a value of $\sim 0.5$ at $E_{I}=0.15 \mathrm{eV}$. Similar to the present study, the translational energy of vibrationally relaxed $\mathrm{H}_{2}$ was higher than that of the vibrationally elastic channel; however, a larger fraction of the relaxation energy is converted to outgoing translation for $\mathrm{H}_{2} / \mathrm{Cu}(0.78-0.90)$ than for $\mathrm{HCl} / \mathrm{Au}(0.26)$.

Attempts have been made to evaluate the importance of electronically nonadiabatic couplings in this system. ${ }^{16,17}$ Electronically adiabatic six-dimensional wave packet calculations of vibrational survival probabilities using an extended PES obtained from density functional theory ${ }^{16}$ are in reasonable agreement with the observed vibrational survival probabilities. However, the adiabatic theory overestimated the probability for rovibrationally inelastic scattering channels where they could be compared to experiment. Despite these differences between experiment and theory, these authors argued $^{16}$ that electron-hole pair excitation was unlikely to be important and that improvements to the PES might lead to better agreement with the experiment. ${ }^{14}$ A nonadiabatic three-dimensional (3D) model was developed by Luntz et al. ${ }^{17}$ which also captured the incidence energy dependence of the survival probability. ${ }^{14}$ Comparison of this model's results to rotationally resolved experiments was not possible since the 3D dynamical model did not describe rotation.

Thus, both adiabatic and nonadiabatic models provide some points of agreement with experiment. This leaves ambiguous the extent to which electronically nonadiabatic effects play an important role in the $\mathrm{H}_{2}(v=1) / \mathrm{Cu}$ collision system. Hence, whether the high outgoing translational energy in $\mathrm{H}_{2}(v=0, J)$ resulting from vibrational relaxation of $\mathrm{H}_{2}(v=1, J=1)$ in collisions with copper provides evidence against the spectator view of electronically nonadiabatic energy transfer or rather reflects electronically adiabatic dynamics remains unclear.

\section{Anticorrelation of translational inelasticity with rotation}

We now return to discuss results of the measurements we have made on various rotational states of the recoiling $\mathrm{HCl}$, presented in Sec. III B and Fig. 11, which shows the dependence of the average $\mathrm{HCl}$ translational energy $\left\langle E_{S}\right\rangle$ on rotational excitation $\Delta E_{\mathrm{rot}}$ induced by the collision with the $\mathrm{Au}$ surface for an incidence energy of $0.52 \mathrm{eV}$. Simply stated, this figure shows that scattering channels with more rotational energy exhibit reduced translational energy, both in the vibrationally elastic (open circles) and inelastic (closed circles) cases. If the energy uptakes by the solid were constant for all rotational channels, this dependence would be one to one. That is, a decrease in $\left\langle E_{S}\right\rangle$ from one $J$-state to another would be perfectly compensated by the excess rotational excitation. However, the reduction in translational energy for channels producing rotationally excited $\mathrm{HCl}$ is incomplete. For example, considering the vibrationally elastic channel, for $J=9$, where more than $0.1 \mathrm{eV}$ additional rotational energy appears in the scattered $\mathrm{HCl}$, we observe only a $0.05 \mathrm{eV}$ reduction in outgoing translational energy. This is a clear evidence of a rotational anticorrelation with translational inelasticity. In other words, the surface excitation accompanying the $\mathrm{HCl}(v=2 \rightarrow 2, J=1 \rightarrow 9)$ channel is 0.05 $\mathrm{eV}$ less than that accompanying the $\operatorname{HCl}(v=2 \rightarrow 2, J=1$ $\rightarrow 1$ ) channel.

To understand this rather subtle effect, consider the following mathematical description. For the elastic channel, the linear relation between outgoing translational and rotational excitation energy is as follows:

$$
\left\langle E_{S}\right\rangle=-\alpha \Delta E_{\text {rot }}+0.23 \text {, }
$$

where energies are in $\mathrm{eV}$.

As the amount of surface excitation, rotational excitation, and final translation energy must sum to the initial incidence energy,

$$
E_{I}=\left\langle E_{S}\right\rangle+\Delta E_{\mathrm{rot}}+E_{\mathrm{surf}},
$$

one may also express the degree of surface excitation in terms of $\Delta E_{\text {rot }}$.

$$
E_{\text {surf }}=E_{I}-\left\langle E_{S}\right\rangle-\Delta E_{\text {rot }}=E_{I}+(\alpha-1) \Delta E_{\text {rot }}-0.23 \text {. }
$$

From this, it is convenient to describe the (anti-)correlation between rotational and surface excitation as a partial derivative,

$$
\frac{\partial E_{\text {surf }}}{\partial \Delta E_{\text {rot }}}=\alpha-1 .
$$

As was mentioned above, if $\alpha=1$, the anticorrelation disappears; that is, the translational inelasticity is independent of rotational excitation. Within this formalism we may also characterize the rotational anticorrelation of translational inelasticity for the vibrationally inelastic channel, also shown in Fig. 11. The numerical results from this analysis are as follows:

$$
\begin{aligned}
\left(\frac{\partial E_{\text {surf }}}{\partial \Delta E_{\text {rot }}}\right)_{\text {elastic }} & =0.48-1 \\
& =-0.52 \text { and }\left(\frac{\partial E_{\text {surf }}}{\partial \Delta E_{\text {rot }}}\right)_{\text {inelastic }} \\
& =0.22-1=-0.78 .
\end{aligned}
$$

In summary, we observe an anticorrelation of translational inelasticity (degree of surface excitation) with rotational excitation. This anticorrelation is stronger for the vibrationally inelastic channel.

An anticorrelation of this type has been observed previously for $\mathrm{NO} / \mathrm{Ag}(111),{ }^{37} \mathrm{NO} / \mathrm{Ge},{ }^{39} \mathrm{~N}_{2} / \operatorname{Ag}(111),{ }^{40}$ and $\mathrm{N}_{2} / \mathrm{Cu}(110)$ (Ref. 41) systems and attributed to the reduction 
in the effective mass of the molecule and hence less efficient translational energy transfer to the solid for those orientations leading to high rotational excitation. ${ }^{37}$ It is possible that a similar explanation is applicable here. However, there is an additional feature of our results that is not anticipated by the previous work on ground state vibrationally elastic scattering. Here we observe the anticorrelation for both vibrationally elastic scattering $(v=2 \rightarrow v=2)$ and for vibrationally inelastic $(v=2 \rightarrow v=1)$ scattering. It appears that the vibrationally inelastic scattering exhibits a stronger anticorrelation than the vibrationally elastic scattering does. A more detailed study of anticorrelation effects in this system would be valuable.

\section{CONCLUSIONS}

We scattered vibrationally excited $\operatorname{HCl}(v=2, J=1)$ from $\mathrm{Au}(111)$ at incidence energies between 0.28 and 1.27 $\mathrm{eV}$. Narrow $\left(\mathrm{FWHM}<30^{\circ}\right)$ angular distributions as well as a strong dependence of final translational energy on initial translational energy show that energy transfer takes place through a direct scattering mechanism. High resolution TOF measurements revealed the translational inelasticity (translation to surface degrees of freedom) for vibrationally elastic $(v=2 \rightarrow 2)$ and inelastic $(v=2 \rightarrow 1)$ channels. For both channels and independent of incidence energy, $\sim 56 \%$ of the incidence energy of translation is transferred to the surface. A significant fraction of the observed recoiling $\mathrm{HCl}$ exhibits translational inelasticity that exceeds the attractive Baule limit. One possible explanation of this observation is that the excitation of electron-hole pairs contributes significantly to the loss of $\mathrm{HCl}$ translational energy in collisions with $\mathrm{Au}(111)$. This suggests that accurate theoretical trapping probabilities for $\mathrm{HCl}$ on $\mathrm{Au}(111)$ may require inclusion of electron-hole pair excitation.

For vibrational relaxation channels, only $26 \%$ of the released vibrational energy appears as outgoing translation, while $74 \%$ couples to the surface. By comparison to previous work where $\operatorname{HCl}(v=2)$ was scattered from an insulator $(\mathrm{MgO})$, we conclude that vibrational relaxation of $\mathrm{HCl}(v$ $=2$ ) on $\mathrm{Au}$ occurs through an electronically nonadiabatic mechanism. Our observations imply that the translational spectator view used to describe electronically nonadiabatic vibrational energy transfer at metal surfaces is only approximately correct. Possible mechanisms by which vibrational energy may be channeled into translation in electronically nonadiabatic vibrational relaxation were described in terms of transient charge transfer.

Taken together, the results obtained in this work provide an important benchmark for future theoretical calculations that attempt to include electronically nonadiabatic influences on energy transfer at surfaces.

\section{ACKNOWLEDGMENTS}

We gratefully acknowledge the financial support from the Department of Energy Office of Basic Energy Sciences, under Grant No. DE-FG02-03ER15441, as well as the Partnership for International Research and Education-for Electronic Chemistry and Catalysis at Interfaces-NSF Grant
No. OISE-0530268.

\section{APPENDIX: CONVERSION OF TOF DATA TO TRANSLATIONAL ENERGY DISTRIBUTIONS}

In order to extract quantitative information about the energy transfer processes upon collision of $\operatorname{HCl}(v=2, J=1)$ with the $\mathrm{Au}(111)$ surface, the measured TOF spectra may be transformed to probability distributions as a function of energy. This appendix describes the method we used to make this transformation.

In REMPI experiments the observed ion signal is proportional to the number density of the particles in the ionization region. Furthermore, a measured REMPI signal within a TOF spectrum, $S(t) d t$, represents the particle density at the detector as a function of time measured over a differential time element. The measured raw TOF data are first converted to flux, $I(t) d t$, by multiplication of the velocity corresponding to each value of $t, v(t)$,

$$
I(t) d t=S(t) v(t) d t .
$$

To transform the time domain data to velocity space, we must conserve probability across each differential time/ velocity element. That is,

$$
P(v) d v=I(t) d t=S(t) v(t) d t,
$$

which leads to

$$
P(v)\left|\frac{d v}{d t}\right|=S(t) v .
$$

The Jacobian is given by

$$
\left|\frac{d v}{d t}\right|=\frac{\ell}{t^{2}}=\frac{v^{2}}{\ell},
$$

where $v$ is the velocity and $l$ is the flight distance. Finally we arrive at the proper transformation,

$$
P(v)=S(t) \frac{l}{v} .
$$

This equation is used to directly invert raw TOF data to velocity space $P(v)$. We then fit this inverted data to a flexible velocity distribution function ${ }^{25}$ of the form given in Eq. (A7). Tables II and III show all of the fitting parameters needed to reproduce the scattered velocity distributions of this work.

The best-fit function obtained in this way as well as the experimentally derived velocity distribution $P(v)$ can then be transformed from velocity to energy space $P(E)$ in a similar way as has just been described. Here, we must recognize that $P(v) d v=P(E) d E$ and employ the Jacobian $d E / d v=m v$ $=\sqrt{2 m E}$,

$$
P(E)=\frac{P(v)}{m v}=\frac{S(t) l}{m v^{2}} .
$$

For completeness we note here that the fitting function displayed in various figures of this article appears in the velocity [Eq. (A7)], time [Eq. (A8)], or energy [Eq. (A9)] domains, 


$$
\begin{aligned}
& P(v)=A v^{3} e^{-\left(\left(v-v_{0}\right) / \alpha\right)^{2}}, \\
& P(t)=A\left(\frac{l}{t}\right)^{4} e^{-(l / \alpha)^{2}\left(1 / t-1 / t_{0}\right)^{2}}, \\
& P(E)=A \frac{E}{m^{2}} e^{-\left(2 / m \alpha^{2}\right)\left(\sqrt{E}-\sqrt{E_{0}}\right)^{2}},
\end{aligned}
$$

where $\mathrm{t}_{0}=v_{0} / l$ and $E_{0}=\frac{1}{2} m v_{0}^{2}$.

${ }^{1}$ A. M. Wodtke, D. Matsiev, and D. J. Auerbach, Prog. Surf. Sci. 83, 167 (2008); M. Silva, R. Jongma, R. W. Field, and A. M. Wodtke, Annu. Rev. Phys. Chem. 52, 811 (2001).

${ }^{2}$ C. T. Rettner, J. Kimman, F. Fabre, D. J. Auerbach, and H. Morawitz, Surf. Sci. 192, 107 (1987).

${ }^{3}$ C. T. Rettner, F. Fabre, J. Kimman, and D. J. Auerbach, Phys. Rev. Lett. 55, 1904 (1985).

${ }^{4}$ A. M. Wodtke, Y. H. Huang, and D. J. Auerbach, J. Chem. Phys. 118 8033 (2003); Y. Huang, A. M. Wodtke, H. Hou, C. T. Rettner, and D. J Auerbach, Phys. Rev. Lett. 84, 2985 (2000); A. M. Wodtke, Y. Huang, and D. J. Auerbach, Chem. Phys. Lett. 413, 326 (2005).

${ }^{5}$ Y. H. Huang, C. T. Rettner, D. J. Auerbach, and A. M. Wodtke, Science 290, 111 (2000).

${ }^{6}$ C. T. Rettner, J. Kimman, and D. J. Auerbach, J. Chem. Phys. 94, 734 (1991).

${ }^{7}$ H. Mortensen, E. Jensen, L. Diekhoner, A. Baurichter, A. C. Luntz, and V. V. Petrunin, J. Chem. Phys. 118, 11200 (2003).

${ }^{8}$ S. A. Adelman and J. D. Doll, J. Chem. Phys. 64, 2375 (1976); J. C. Tully, ibid. 73, 1975 (1980); R. W. Zwanzig, ibid. 32, 1173 (1960).

${ }^{9}$ Q. Ran, D. Matsiev, D. J. Auerbach, and A. M. Wodtke, Nucl. Instrum. Methods Phys. Res. B 258, 1 (2007).

${ }^{10}$ B. D. Kay, T. D. Raymond, and M. E. Coltrin, Phys. Rev. Lett. 59, 2792 (1987).

${ }^{11}$ Q. Ran, D. Matsiev, D. J. Auerbach, and A. M. Wodtke, Phys. Rev. Lett. 98, 237601 (2007).

${ }^{12}$ D. M. Newns, Surf. Sci. 171, 600 (1986).

${ }^{13}$ M. Gostein, E. Watts, and G. O. Sitz, Phys. Rev. Lett. 79, 2891 (1997).

${ }^{14}$ E. Watts and G. O. Sitz, J. Chem. Phys. 114, 4171 (2001).

${ }^{15}$ L. C. Shackman and G. O. Sitz, J. Chem. Phys. 123, 064712 (2005).

${ }^{16}$ E. Watts, G. O. Sitz, D. A. McCormack, G. J. Kroes, R. A. Olsen, J. A. Groeneveld, J. N. P. Van Stralen, E. J. Baerends, and R. C. Mowrey, J. Chem. Phys. 114, 495 (2001).

${ }^{17}$ A. C. Luntz, M. Persson, and G. O. Sitz, J. Chem. Phys. 124, 091101 (2006).

${ }^{18}$ K. R. Lykke and B. D. Kay, J. Chem. Phys. 92, 2614 (1990).

${ }^{19}$ Q. Ran, D. Matsiev, A. M. Wodtke, and D. J. Auerbach, Rev. Sci. Instrum. 78, 104104 (2007)

${ }^{20}$ D. S. Green, G. A. Bickel, and S. C. Wallace, J. Mol. Spectrosc. 150, 354 (1991); D. S. Green, G. A. Bickel, and S. C. Wallace, ibid. 150, 388 (1991); D. S. Green, G. A. Bickel, and S. C. Wallace, ibid. 150, 303
(1991); D. S. Green and S. C. Wallace, J. Chem. Phys. 96, 5857 (1992).

${ }^{21}$ B. Baule, Ann. Phys. 349, 145 (1914); A. Zangwill, Physics at Surface (Cambridge University Press, Cambridge, 1988).

${ }^{22}$ J. Harris, in Dynamics of Gas-Surface Interactions, edited by C. T. Rettner and M. N. R. Ashfold (Royal Society of Chemistry, Letchworth, 1991), p. 1

${ }^{23}$ F. O. Goodman and H. Y. Wachman, Dynamics of Gas-Surface Scattering (Academic Press, New York, 1976).

${ }^{24}$ The modified Baule equation that takes into account the thermal motion of the gold atoms results in a negligible change to this line of argumentation.

${ }^{25}$ J. E. Hurst, L. Wharton, K. C. Janda, and D. J. Auerbach, J. Chem. Phys. 78, 1559 (1983).

${ }^{26}$ J. E. Hurst, L. Wharton, K. C. Janda, and D. J. Auerbach, J. Chem. Phys. 83, 1376 (1985).

${ }^{27}$ J. A. Barker and D. J. Auerbach, Surf. Sci. Rep. 4, 1 (1984); E. W. Kuipers, M. G. Tenner, M. E. M. Spruit, and A. W. Kleyn, Surf. Sci. 205, 241 (1988).

${ }^{28}$ M. E. M. Spruit, P. J. Vandenhoek, E. W. Kuipers, F. H. Geuzebroek, and A. W. Kleyn, Surf. Sci. 214, 591 (1989).

${ }^{29}$ K. D. Gibson, S. J. Sibener, H. P. Upadhyaya, A. L. Brunsvold, J.-M. Zhang, T. K. Minton, and D. Troya, J. Chem. Phys. 128, 224708 (2007).

${ }^{30}$ Y. Watanabe, H. Yamaguchi, M. Hashinokuchi, K. Sawabe, S. Maruyama, Y. Matsumoto, and K. Shobatake, Eur. Phys. J. D 38, 103 (2006).

${ }^{31}$ M. Korolik, M. M. Suchan, M. J. Johnson, D. W. Arnold, H. Reisler, and C. Wittig, Chem. Phys. Lett. 326, 11 (2000).

${ }^{32}$ L. C. Shackman and G. O. Sitz, J. Chem. Phys. 123, 064712 (2005).

${ }^{33} \mathrm{Here}$, we used the covalent and van der Waals radii of $\mathrm{Au}$ and $\mathrm{Cl}$ atoms.

${ }^{34}$ The additional factor of 2 is included to reflect the fact that the Debye frequency represents the highest frequency, thus $\omega_{D} / 2$ represents a phonon frequency in the middle of the phonon energy spectrum.

${ }^{35}$ A. Amirav and M. J. Cardillo, Phys. Rev. Lett. 57, 2299 (1986); A. Danon, A. Vardi, and A. Amirav, ibid. 65, 2038 (1990).

${ }^{36}$ J. Misewich and M. M. T. Loy, J. Chem. Phys. 84, 1939 (1986); J. Misewich, H. Zacharias, and M. M. T. Loy, Phys. Rev. Lett. 55, 1919 (1985).

${ }^{37}$ J. Kimman, C. T. Rettner, D. J. Auerbach, J. A. Barker, and J. C. Tully, Phys. Rev. Lett. 57, 2053 (1986).

${ }^{38}$ M. F. Somers, D. A. McCormack, G. J. Kroes, R. A. Olsen, E. J. Baerends, and R. C. Mowrey, J. Chem. Phys. 117, 6673 (2002); D. A. McCormack, G. J. Kroes, R. A. Olsen, J. A. Groeneveld, J. N. P. van Stralen, E. J. Baerends, and R. C. Mowrey, Faraday Discuss. 117, 109 (2000) c38 c38a c38b c38cD. A. McCormack, G. J. Kroes, R. A. Olsen, J. A. Groeneveld, J. N. P. van Stralen, E. J. Baerends, and R. C. Mowrey, Chem. Phys. Lett. 328, 317 (2000).

${ }^{39}$ F. Budde, A. Modl, A. V. Hamza, P. M. Ferm, and G. Ertl, Surf. Sci. 192, 507 (1987).

${ }^{40}$ G. O. Sitz, A. C. Kummel, and R. N. Zare, J. Chem. Phys. 89, 2558 (1988).

${ }^{41}$ J. L. W. Siders and G. O. Sitz, J. Chem. Phys. 101, 6264 (1994). 